OPEN ACCESS

Edited by:

Zhichao Fan,

UCONN Health, United States

Reviewed by:

Rongrong Liu,

Northwestern University,

United States

Bo Liu,

University of California, Berkeley,

United States

*Correspondence:

Sarah Y. Yuan

syuan@usf.edu

Specialty section:

This article was submitted to Cell Adhesion and Migration,

a section of the journal

Frontiers in Cell and Developmental

Biology

Received: 29 August 2020

Accepted: 30 September 2020

Published: 28 October 2020

Citation:

Yang $X$, Chatterjee $V$, Ma Y, Zheng E and Yuan SY (2020) Protein Palmitoylation in Leukocyte Signaling

and Function.

Front. Cell Dev. Biol. 8:600368. doi: $10.3389 /$ fcell.2020.600368

\section{Protein Palmitoylation in Leukocyte Signaling and Function}

\author{
Xiaoyuan Yang ${ }^{1}$, Victor Chatterjee ${ }^{1}$, Yonggang $\mathrm{Ma}^{1}$, Ethan Zheng ${ }^{1}$ and Sarah Y. Yuan ${ }^{1,2 *}$ \\ ${ }^{1}$ Department of Molecular Pharmacology and Physiology, Morsani College of Medicine, University of South Florida, Tampa, \\ FL, United States, ${ }^{2}$ Department of Surgery, Morsani College of Medicine, University of South Florida, Tampa, FL, \\ United States
}

Palmitoylation is a post-translational modification (PTM) based on thioester-linkage between palmitic acid and the cysteine residue of a protein. This covalent attachment of palmitate is reversibly and dynamically regulated by two opposing sets of enzymes: palmitoyl acyltransferases containing a zinc finger aspartate-histidine-histidine-cysteine motif (PAT-DHHCs) and thioesterases. The reversible nature of palmitoylation enables fine-tuned regulation of protein conformation, stability, and ability to interact with other proteins. More importantly, the proper function of many surface receptors and signaling proteins requires palmitoylation-meditated partitioning into lipid rafts. A growing number of leukocyte proteins have been reported to undergo palmitoylation, including cytokine/chemokine receptors, adhesion molecules, pattern recognition receptors, scavenger receptors, T cell co-receptors, transmembrane adaptor proteins, and signaling effectors including the Src family of protein kinases. This review provides the latest findings of palmitoylated proteins in leukocytes and focuses on the functional impact of palmitoylation in leukocyte function related to adhesion, transmigration, chemotaxis, phagocytosis, pathogen recognition, signaling activation, cytotoxicity, and cytokine production. Keywords: palmitoylation, protein function, leukocyte function/activation, signal transduction, DHHC palmitoyl
transferases, leukocyte behaviors

\section{INTRODUCTION}

Leukocytes are critical components of innate and adaptive immunity by eradicating microbes and potentially harmful cells or substances. In addition to combating infection, leukocytes are also involved in the pathogenesis of many diseases, including cancer, neurological disorders, and cardiovascular diseases. In order to ensure effective leukocyte function without causing unwanted damages to normal tissues, the cascades of leukocyte responses are precisely controlled by many leukocyte proteins such as adhesion molecules, surface receptors, co-receptors, signaling effectors, adaptor proteins, cytokines, and chemokines (Yadav et al., 2003). Aberrant function of any of these proteins may lead to abnormal immune responses. Leukocyte proteins undergo a variety of posttranslational modifications (PTMs) to ensure fine-tuned regulation and functional diversity. These modifications include acylation, phosphorylation, ubiquitination, glycosylation, nitrosylation, methylation, and proteolysis (Liu et al., 2016). Among these PTMs, protein palmitoylation is a prominent type of acylation that has gained increasing recognition for its roles in regulating leukocyte signaling and behaviors. 
Palmitoylation belongs to a subtype of fatty acid modification called S-acylation, involving covalent attachment of 16-carbon palmitic acid to one or more cysteine residues of a protein through a thioester linkage (R-S-CO-R'). In 1979, palmitoylation was first identified by Schmidt and Schlesinger (1979). The source of palmitic acid is the cytosolic pool of palmitoyl-CoA (Resh, 2006a). The addition of palmitic acid is an enzyme-catalyzed reaction (Blaskovic et al., 2013). Palmitoylation differs from other types of lipid modifications (e.g., myristoylation and prenylation) in that palmitoylation is reversible and dynamically regulated. The attached palmitate is readily removed when the thioester linkage is cleaved. Cycles of palmitoylation and de-palmitoylation are precisely regulated in a fashion similar to phosphorylation and ubiquitination. The reversibility of palmitoylation allows for the dynamic regulation and fine-tuning of protein function and activities in leukocytes.

Although protein palmitoylation was discovered about 40 years ago, the field has substantially progressed only in recent years after the breakthrough of non-radioactive detection of palmitoylated proteins. Palmitoylation has since been extensively studied in cancer and neurological disorders (Kang et al., 2008; Ko and Dixon, 2018); however, research on palmitoylation regulation in leukocyte function is in its infancy, with many molecular mechanisms remaining largely unexplored. Recently, methodological advances have made it possible to perform sensitive and reliable large-scale palmitoyl proteome profiling in different subtypes of leukocytes, including monocyte/macrophages, dendritic cells, T cells and B cells, as well as in cells directly interacting with leukocytes, such as endothelial cells (ECs; Martin and Cravatt, 2009; Merrick et al., 2011; Ivaldi et al., 2012; Marin et al., 2012; Chesarino et al., 2014). These palmitoyl proteomic approaches have identified an array of palmitoylated proteins, including but not limited to cytokine/chemokine receptors CCR5, TNFR, and INFAR; adhesion molecules PECAM-1 and JAM-C; pattern recognition TLR/MYD88 complex; scavenger receptor CD36; T cell coreceptors CD4 and CD8; Src family kinases Lck and Fyn; transmembrane adaptor proteins linker of activated T cells (LAT) and PAG; signaling effectors $\mathrm{G} \alpha$, Ras, and phospholipase C; and $\mathrm{Ca}^{2+}$ regulator IP3R. This growing list of palmitoylated proteins highlights the functional significance of palmitoylation in leukocytes. In this review, we discuss the latest advances in palmitoylation-mediated regulation of protein function and its impact on leukocyte signaling and behaviors.

\section{PALMITOYLATION IN PROTEIN FUNCTION AND SIGNAL TRANSDUCTION}

The regulatory mechanisms of palmitoylation in protein function and signal transduction are summarized in Figure 1.

\section{Subcellular Effects}

Covalent attachment of palmitic acid to proteins results in increased protein hydrophobicity and, therefore, affects protein function mainly through four mechanisms: altering conformation/structure, directing intracellular trafficking or compartmentalization, regulating stability/half-life, and influencing protein-protein interactions. Firstly, palmitoylation plays a critical role in regulating protein conformation. Many G-protein coupled receptors (GPCRs) in leukocytes require palmitoylation for their proper conformation. The insertion of the attached palmitate into plasma membrane creates the fourth loop of GPCRs between the cytoplasmic end of the seventh $\alpha$-helix and palmitate insertion, which is essential for the propagation of GPCR signals (Chini and Parenti, 2009). Secondly, palmitoylation regulates protein trafficking and localization, as typically seen with small GTPase Ras, one of the most studied palmitoylated substrates. The palmitoylation and de-palmitoylation cycle dynamically regulates Ras shuttling between Golgi and plasma membrane (Hornemann, 2015). Likewise, many transmembrane or membrane-associated proteins rely on palmitoylation for their stable membrane anchor (Greaves and Chamberlain, 2007). Thirdly, emerging evidence has shown that palmitoylation can stabilize proteins and increase their half-life. Mutation of the palmitoylation site greatly shortens the life span of many proteins, as such proteins undergo misfolding during protein synthesis or become more susceptible to ubiquitination-mediated degradation (Resh, 2006a). Finally, palmitoylation modulates protein-protein interactions both directly and indirectly. The long hydrocarbon chain of the attached palmitic acid can interact directly with that of another protein. Palmitoylation also promotes the clustering of proteins in specialized membrane microdomains, which selectively brings specific proteins into proximity and facilitates their interactions indirectly (Blaskovic et al., 2013).

\section{Impact in Signal Transduction}

A growing list of receptors and signaling proteins in leukocytes have been recognized as palmitoylation substrates (Resh, 2006a). Their function can be modulated by palmitoylation in similar manners as stated above. Additionally, palmitoylation facilitates the sequestering of receptors, signaling proteins, and adaptor proteins into lipid rafts for effective signal transduction (Bijlmakers and Marsh, 2003). Lipid rafts are liquid-ordered microdomains in the external leaflet of plasma membrane enriched with cholesterol and glycosphingolipids; they are highly dynamic and serve as a platform for many signal transduction processes in leukocytes (Alonso and Millan, 2001). For example, the signaling proteins for $\mathrm{T}$-cell activation, including the $\mathrm{T}$ cell receptor, its co-receptors (CD4 and CD8), Src-family kinases (Lyn and Fyn), and adaptor proteins are all clustered in lipid rafts of $\mathrm{T}$ cells (Kabouridis, 2006). As a saturated fatty acid, palmitate prefers to insert into liquid-ordered raft domains rather than the bulk plasma membrane (Resh, 2013). Palmitoylation-dependent segregation of proteins in lipid rafts is essential for the amplification of signal propagation in activated cells while preventing the signal activation in resting cells. Another unique function of palmitoylation in regulating signal transduction is mediating receptor desensitization and internalization (Resh, 2006a). Upon activation, many receptors become desensitized as a result of phosphorylation mediated by GPCR kinases (GRKs), the function of which is greatly 


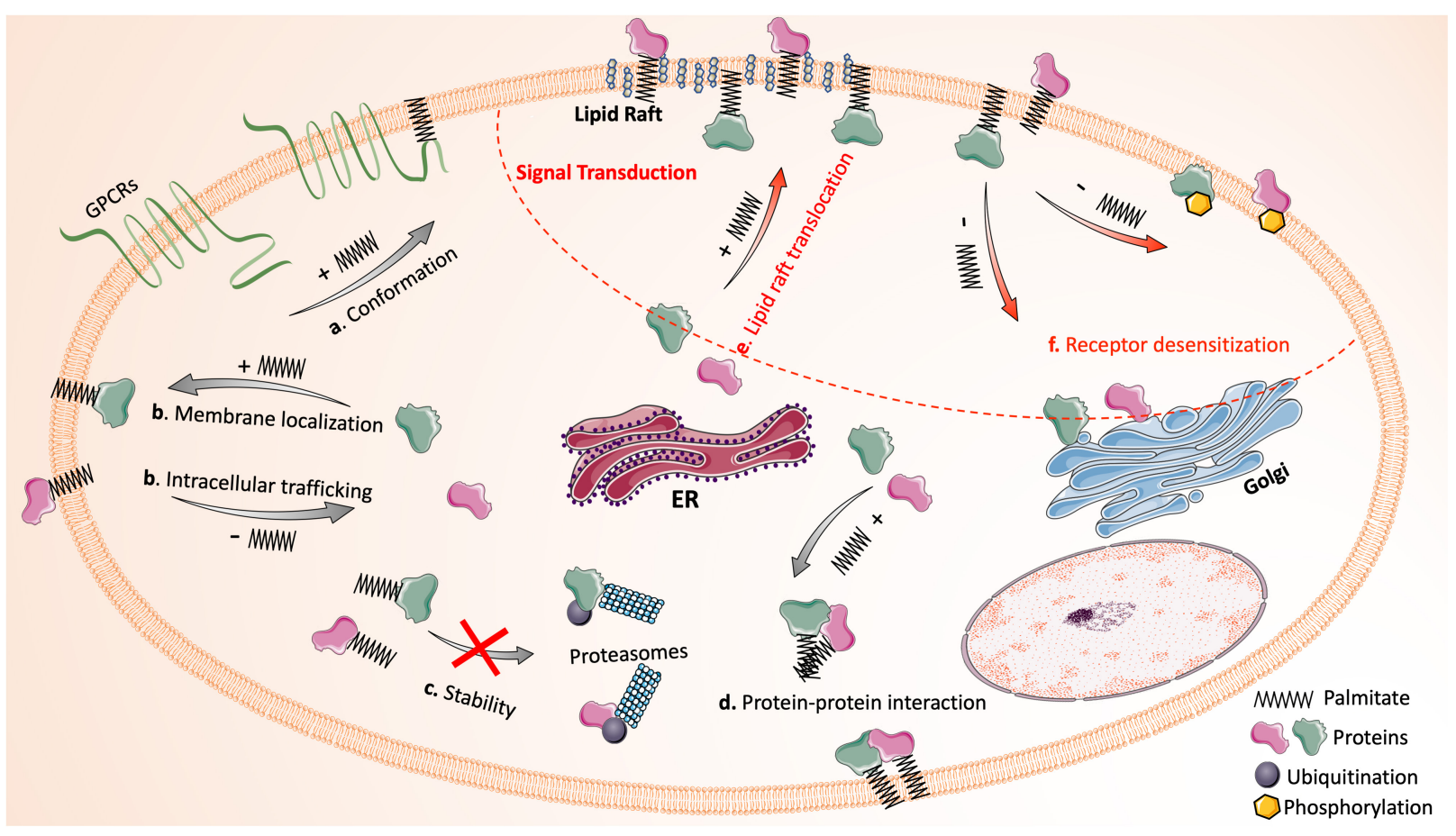

FIGURE 1 | Protein palmitoylation dynamically regulates protein function and signal transduction. The reversible attachment of palmitic acid affects protein function in many ways: (a) palmitoylation alters the conformation or structure of a protein (e.g., the fourth intracellular loop of GPCRs that is formed by inserting palmitate into the plasma membrane); (b) palmitoylation mediates protein intracellular trafficking and ensures stable membrane localization of proteins; (c) palmitoylation stabilizes proteins by preventing misfolding and ubiquitination-mediated degradation; (d) palmitoylation facilitates protein-protein interaction directly and indirectly. In addition, the palmitoylation and de-palmitoylation cycle has critical impact on signal transduction (upper right region circled by red dash line): (e) many signaling proteins require palmitoylation for their translocation into lipid rafts. (f) De-palmitoylation mediates receptor desensitization following activation by promoting the phosphorylation of receptors or the translocation of membrane signaling proteins to cytoplasm. Images of proteins and organelles were obtained from Smart Servier Medical Art (https://smart.servier.com).

enhanced by palmitoylation (Stoffel et al., 1998). Meanwhile, de-palmitoylation of receptors has been proved to increase the accessibility of their phosphorylation sites to kinases (Moffett et al., 1993; Qanbar and Bouvier, 2003). Thus, palmitoylation and de-palmitoylation precisely control signaling events in a spatialtemporal context, regulating many aspects of leukocyte function.

As the fundamental signaling events in many leukocytes, GPCR signal transduction is subjected to palmitoylationdependent regulation at many levels. In addition to GPCRs themselves being affected by palmitoylation in the aforementioned manner, various GPCR downstream effectors are also substrates of palmitoylation. Upon GPCR activation, $\mathrm{G} \alpha$ subunit dissociates from $\mathrm{G} \beta \gamma$ subunit, and their fragments subsequently activate three major signal transduction cascades: (1) Adenylate cyclase cascade, which catalyzes the conversion of ATP to cAMP and then activates PKA and its downstream effectors; (2) Phospholipase c (PLC) cascade, which promotes the conversion of phosphatidylinositol 4,5-bisphosphate [PI(4,5)P2] to diacylglycerol (DAG) and inositol 1,4,5-trisphospate (IP3). IP3 then binds to IP3 receptor (IP3R) localized on ER and induces the release of $\mathrm{Ca}^{2+}$ into cytoplasm. DAG and increased $\mathrm{Ca}^{2+}$ are both capable of activating PKC; (3) Phosphatidylinositol 3 kinase (PI3K) cascade, which hydrolyzes PI(4,5)P2 to produce phosphatidylinositol 3,4,5-triphosphate (PIP3), an effective activator of AKT (also known as PKB; Hilger et al., 2018; Lammermann and Kastenmuller, 2019; Wang X. et al., 2019). Many effector proteins of the GPCR signaling cascades, for example, G $\alpha$, PLC and IP3R, require palmitoylation for their proper localization and function. $\mathrm{G} \alpha \mathrm{q}$ is palmitoylated at Cys9 and 10, while Gas is palmitoylated at Cys3 (Wedegaertner

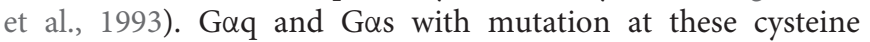
sites exist in their soluble form in cytoplasm rather than their primary location in lipid rafts. Palmitoylation-defective G $\alpha \mathrm{q}$ and Gas are incapable of coupling with GPCRs and fail to induce the activation of PLC or adenylate cyclase, respectively (Wedegaertner et al., 1993). A separate study shows that the palmitate turnover rate of $\mathrm{G} \alpha$ increases upon $\mathrm{G} \alpha$ activation and dissociation from $\mathrm{G} \beta \gamma$; de-palmitoylation mediates the internalization of $\mathrm{G} \alpha$ to cytoplasm, where it is repalmitoylated (Ross, 1995). This activation-dependent de-palmitoylation of $\mathrm{G} \alpha$ is essential to the desensitization process following GPCR activation (Vogler et al., 2008).

Ras, which belongs to the small GTPases, also functions as a signaling effector. Ras is one of the earliest identified palmitoylation substrates (Magee et al., 1987). All three Ras isoforms $(\mathrm{H}-, \mathrm{N}$-, and $\mathrm{K}-\mathrm{Ras})$ undergo farnesylation at the C-terminal CAAX motif, allowing Ras to loosely insert into endomembrane structures. N-Ras and H-Ras, but not K-Ras, 
are further modified by attaching palmitates at one or two cysteines (Cys181 in N-Ras, Cys181 and 184 in H-Ras) adjacent to CAAX motif in Golgi (Hancock et al., 1990; Swarthout et al., 2005; Baumgart et al., 2010). The attached palmitate promotes the translocation of Ras to plasma membrane and facilities the stable membrane anchor of Ras. Reports show that palmitoylation-defective $\mathrm{H}$-Ras is trapped in Golgi and unable to traffic to plasma membrane. Palmitoylation also influences the GTP-binding ability of Ras. Functional assay revealed that only palmitoylated N-Ras turns into the GTP-bound activated form in response to agonists (Song et al., 2013). The palmitate turnover rate of GTP-bound activated Ras is 25-fold higher than that of GDP-bound deactivated Ras (Baker et al., 2003). After de-palmitoylation, Ras traffics to Golgi, where it is palmitoylated again and redirected to plasma membrane for the next cycle.

\section{PALMITOYLATION ENZYMES}

The turnover of protein palmitoylation is generally much faster than the turnover of the particular protein being palmitoylated, indicating that the attachment of palmitate is dynamically regulated (Won et al., 2018). Protein palmitoylation is controlled by two opposing types of enzymes. Palmitoyl acyltransferases (PATs) catalyze the addition of palmitic acid, while the removal of palmitic acid is mediated by thioesterases, including palmitoyl protein thioesterases (PPTs), acyl-protein thioesterases (APTs) and $\alpha / \beta$ hydrolase domain-containing 17 proteins $(\mathrm{ABHD} 17 \mathrm{~s}$; Won et al., 2018). Under the tight control of these enzymes, the palmitoylation and de-palmitoylation cycle normally occurs multiple times throughout the lifetime of a palmitoylated protein.

\section{Palmitoyl Acyltransferases}

Palmitoyl acyltransferases contain a highly conserved Asp-HisHis-Cys (DHHC) tetrapeptide motif in its cysteine-rich domain; thus these enzymes are also known as DHHCs or PAT-DHHCs (Tabaczar et al., 2017). DHHCs were first identified by Deschenes and Davis labs in 2002 (Lobo et al., 2002; Roth et al., 2002). So far, 23 DHHCs have been identified in humans (Rana et al., 2019). DHHCs are transmembrane proteins that span the membrane four to six times and locate on the cytosolic face of the membrane (Blaskovic et al., 2013). The conserved cysteine-rich domain serves as the catalytic center for the enzyme, directly involved in the palmitoyl transfer reaction (Tabaczar et al., 2017; Rana et al., 2019). The majority of DHHCs are localized in endoplasmic reticulum (ER; DHHC1, 6, 14, 19, 23, 24) or Golgi apparatus (DHHC 3, 4, 7, 8, 15, 17, 18) or both (e.g., DHHC 2, 9, 12, 13, 22), while several others (e.g., DHHC 5, 20, 21) can be found on plasma membrane (Korycka et al., 2012; Hornemann, 2015; Ko and Dixon, 2018). DHHCs catalyze palmitoylation in a two-step manner: (1) auto-palmitoylation reaction where the cysteine residue in DHHC motif binds with palmitoyl-CoA; (2) acyl transfer reaction where the palmitoyl group is transferred from DHHCs to cysteine residues of a protein substrate (Tabaczar et al., 2017). To date, the substrate specificity of DHHCs is poorly understood. Although the palmitoylated cysteines share some common characteristics, there is no consensus palmitoylation sequence (Salaun et al., 2010). It is speculated that the proteinprotein interaction domain of DHHCs (e.g., ankyrin repeats in DHHC13/17, Src-homology 3 domain in DHHC6) plays critical roles in recognizing specific substrates (Fredericks et al., 2014; Verardi et al., 2017; Matt et al., 2019).

Accumulating studies have revealed the functional impact of DHHCs in leukocytes. For example, mice with DHHC21 deficiency display a reduced number of slow-rolling and adherent leukocytes in post-capillary venules during systemic inflammation (Beard et al., 2016). There is evidence for DHHC6- catalyzed MyD88 palmitoylation in neutrophils, which is necessary for TLR-NF- $\kappa \mathrm{B}$ signaling (Kim et al., 2019). In dendritic cells, surface expression and proper function of TLR2 depend on the palmitoylation of TLR2 by DHHC 2, 3, 6, 7, or 15 (Chesarino et al., 2014). DHHC7 is responsible for the palmitoylation of Fas, thus regulating $\mathrm{T}$ cell apoptosis and differentiation (Rossin et al., 2015). Although beyond the current focus, it is worth pointing out that palmitoylation can affect the infectivity of various viruses and bacteria, including coronavirus, HIV, influenza, and B anthrax (Sobocinska et al., 2017). It has been reported that DHHC-mediated palmitoylation of the SARS$\mathrm{CoV}$ spike protein facilitates the virus entry into host cells (Petit et al., 2007). This may also hold true for the SARS-Cov2 virus, as a study has reported the interaction of SARS-Cov2 with DHHC5 (Gordon et al., 2020).

\section{De-Palmitoylation Enzymes}

Many signaling proteins in leukocytes, such as Ras and certain $\mathrm{G}$ protein subunits, undergo rapid de-palmitoylation upon activation, suggesting that de-palmitoylation enzymes participate in leukocyte responses (Won et al., 2018). So far, three classes of de-palmitoylation enzymes have been identified: PPTs, APTs and ABHD17s. PPTs (PPT1 and PPT2) mainly reside in lysosomes and mediate the removal of palmitate during the process of protein degradation (Resh, 2006a; Koster and Yoshii, 2019). PPT1 was the first identified enzyme that removes palmitate from a modified protein (Camp and Hofmann, 1993). Although PPT1 has been reported to show activities outside of lysosomes in certain neurons, it is unlikely that PPT1 contributes to the depalmitoylation of cytoplasmic or plasma membrane proteins (Kim et al., 2008; Hornemann, 2015). PPT2 shares $18 \%$ of its amino acid sequence with PPT1 and shows a certain degree of cross-reactivity with PPT1 (Gupta et al., 2001; Kim et al., 2008; Cho and Park, 2016). However, PPT2 does not catalyze the de-palmitoylation of H-Ras as PPT1 does (Soyombo and Hofmann, 1997), suggesting a distinctive substrate specificity for PPT2. APTs (APT1 and APT2) are ubiquitously expressed serine hydrolases, localized predominantly in cytoplasm (Kong et al., 2013). APT1 (or LYPLA1) was first identified as a depalmitoylation enzyme in 1998, based on its ability to remove $\left[{ }^{3} \mathrm{H}\right]$ palmitate from palmitoylated $\mathrm{G} \alpha$ and $\mathrm{H}$-Ras (Duncan and Gilman, 1998). Further studies show that APT1 catalyzes depalmitoylation of numerous leukocyte proteins, including linker of activated $\mathrm{T}$ cells (LAT), endothelial nitric oxide synthase (eNOS), regulator of G protein signaling protein (RGS), and synaptosome associated protein 23 (SNAP-23; Ladygina et al., 2011). APT2 exhibits $68 \%$ of amino acid sequence similarity to 
APT1 and has an almost identical 3D-structure to APT1 (Gadalla and Veit, 2020). APT2 seems to possess distinct substrate preference compared to APT1. Nevertheless, APT2 and APT1 are functionally redundant in de-palmitoylating certain proteins (e.g., Ras; Rusch et al., 2011). So far, little is known about the mechanism of APT substrate specificity. Several studies have investigated the regulatory mechanism and functional impact of APTs in leukocytes. The expression of APT1 in RAW264.7 macrophage-like cells decreases in response to LPS, while the expression of APT2 remains unaffected (Satou et al., 2010). $A$ recent study of $B$ cells shows that APTs are inhibited by miR138 and -424 , which exerts critical impacts on B cell apoptosis in a CD95-dependent mechanism (Berg et al., 2015). ABHD17s (ABHD17A, ABHD17B and ABHD17C) are mainly localized to plasma membrane of various cell types (Lin and Conibear, 2015; Won et al., 2018). The ABHD17s were first identified as the de-palmitoylase for N-Ras (Lin and Conibear, 2015). ABHD17s are palmitoylated at the $\mathrm{N}$-terminal cysteine cluster, which is required for its plasma membrane localization and substrate interaction (Lin and Conibear, 2015). So far, there has been limited research to characterize the functional impacts and regulatory mechanisms of $\mathrm{ABHD} 17 \mathrm{~s}$.

\section{METHODS OF DETECTING PALMITOYLATION}

Detection of palmitoylated protein has been technically challenging due to its reliance on time-consuming radioactive labeling. Recently, several methodological breakthroughs have been achieved for quantifying and imaging palmitoylated proteins, which greatly accelerate the research progress in this field. Currently, there are three major types of detection methods: radioactive metabolic incorporation, hydroxylamine-based acylexchange assay, and click chemistry-based metabolic labeling. All can be applied to detect palmitoylated proteins in leukocytes.

\section{Radioactive Metabolic Incorporation}

Palmitoylated proteins can be metabolically labeled with palmitic acid incorporated with a radioisotope. Tritiated palmitate (or $\left[{ }^{3} \mathrm{H}\right]$ palmitate) is the most commonly used radioactive palmitate as it is commercially available and confirmed as reliable by many labs. This method consists of four steps: $\left[{ }^{3} \mathrm{H}\right]$ palmitate labeling, immunoprecipitation proteins, SDS-PAGE, and photofluorographic exposure (Resh, 2006b). When coupling it with a pulse-chase approach, $\left[{ }^{3} \mathrm{H}\right]$ palmitate labeling can be utilized to study the palmitate turnover of a specific protein substrate. For more than two decades, radioactive labeling has remained the gold standard for detecting palmitoylation (Yount et al., 2013). However, $\beta$ particles emitted by $\left[{ }^{3} \mathrm{H}\right]$ are weak, thus it often requires several weeks to develop a clear $\mathrm{X}$-ray image through photofluorography. The time-consuming and cumbersome nature of this method limits its use in many research labs, not to mention the hazards associated with handling radioactive materials. Later, a more energetic radioactive palmitate analog $\left[{ }^{125} \mathrm{I}\right]$ iodohexadecanoic acid $\left({ }^{125} \mathrm{I}\right.$ IC16) was adapted to this method. $\left[{ }^{125} \mathrm{I}\right]$ generates gamma rays that are more penetrating, thus greatly shortening the exposure time to $24 \mathrm{~h}$ (Tsai et al., 2014). Other advances in radioactive labeling involve improving the sensitivity of $\mathrm{X}$-ray films, which enables visualization of $\left[{ }^{3} \mathrm{H}\right]$ palmitate-labeled proteins in 1-3 days (Tsai et al., 2014).

\section{Hydroxylamine-Based Acyl-Exchange Assay}

Hydroxylamine-based acyl-exchange assay is one of the two nonradioactive techniques invented to circumvent the drawbacks of radioactive labeling (Drisdel and Green, 2004). This method is comprised of four biochemical steps: (1) blocking the free thiols of extracted proteins; (2) cleaving the thioester linkage with hydroxylamine to liberate the previously palmitoylated cysteinyl thiols; (3) capturing the newly freed thiol groups with pyridyldithiol-biotin; (4) purifying the biotin-labeled proteins using streptavidin agarose beads (Wan et al., 2007; Brigidi and Bamji, 2013). More recently, acyl-biotin exchange assay (ABE) was modified to a simplified version called resin-assisted capture (RAC) in which proteins with hydroxylamine-liberated thiol groups are directly captured by pyridyldithiol-resin (Forrester et al., 2011). The acyl-exchange assays are more powerful when coupled with mass spectrometry-based proteomic studies. Unlike radioactive labeling that analyzes a single protein, acyl-exchange assays enable large-scale profiling of the proteome, leading to the discovery of numerous novel palmitoylation substrates in leukocytes (Ivaldi et al., 2012). Moreover, acyl-exchange assays are unique among all the palmitoylation detecting methods for their capability in providing a snapshot of the palmitoylated protein profile in cells. The major disadvantage of acyl-exchange assays lies within the fact that they are not compatible with pulse-chase experiments, as the entire acyl-exchange procedure is performed in protein lysates (Tsai et al., 2014).

\section{"Click Chemistry"-Based Metabolic Labeling}

"Click chemistry"-based metabolic labeling is the second type of non-radioactive techniques used to detect palmitoylation. The principle behind this method is the "click chemistry" reaction, also known as $\mathrm{Cu}^{1+}$-catalyzed Huisgen cycloaddition reaction between azido- and alkynyl-groups (Hang et al., 2007; Charron et al., 2009; Hannoush and Arenas-Ramirez, 2009). The most commonly used alkynyl-palmitate analogs are $\omega$-alkynylC16 (Alk-C16 or 15-hexadecynoic acid) and 17-octadecynoic acid (17-ODYA). "Click chemistry"-based metabolic labeling is most versatile in application compared to other methods due to the various types of azido-containing markers. First and foremost, palmitoylated proteins labeled with alkynyl groups can react with azido-fluorescent dye (e.g., Oregon Green 488 azide), which enables the robust imaging of global protein palmitoylation in cells (Hannoush and Arenas-Ramirez, 2009; Beard et al., 2016). Later, this imaging strategy was further improved by coupling with proximity ligation assay, which makes it possible to visualize a single palmitoylated protein and study its palmitoylation level and subcellular localization (Gao and Hannoush, 2014). Secondly, alkynyl-palmitoylated proteins 
can also react with azido-biotin for streptavidin enrichment, followed by either single protein analysis or large-scale LCMS/MS proteomic analysis in leukocytes (Ladygina et al., 2011). Thirdly, alkynyl-palmitoylated proteins can bind to azido-IRDye 800 , omitting the streptavidin enrichment step and allowing the direct detection of leukocyte palmitoylated proteins using Li-COR infrared imaging (Akimzhanov and Boehning, 2015). However, alkynyl-palmitate analogs can only incorporate into accessible and reduced cysteine residues of a palmitoylated protein. Stalely palmitoylated proteins, which do not undergo dynamic palmitate turnover, cannot be labeled or purified by this method as their cysteines are not available to conjugate with alkynyl-palmitate analogs.

\section{Method to Identify Palmitoylation Sites}

The palmitoylation sites of the modified protein can be predicted by CSS-Palm 2.0 using a clustering and scoring strategy algorithm, or by other tools like GPS-lipid. To verify the predicted cysteine residues, mutagenesis of cysteine residue(s) is performed, often followed by studying the subsequent impacts on protein palmitoylation level, subcellular localization, stability, and activity. Cysteine residue of a palmitoylated protein is often substituted with alanine, though cysteine-to-serine mutagenesis is also employed by some research groups.

\section{PROTEIN PALMITOYLATION REGULATION OF LEUKOCYTE FUNCTION}

A growing number of palmitoylated proteins have been discovered in leukocytes, many of which play critical roles in regulating various leukocyte functions, including adhesion, transmigration, chemotaxis, phagocytosis, pathogen recognition, T cell receptor (TCR) and BCR signaling activation, cytotoxicity, cytokine production, and apoptosis. Below, we discuss the functional impact of palmitoylation in different types of leukocytes (Figure 2).

\section{Neutrophils}

Neutrophils are the most abundant type of leukocytes in human circulation that act as ongoing surveillance against potential invading pathogens and sterile inflammation. They are among the first responders to sites of infection and contribute to the initiation and propagation of inflammatory cascades (Ma et al., 2019). The neutrophil recruitment cascade is initiated with selectin-mediated slow rolling, followed by $\beta 2$-integrin-mediated firm adhesion and PECAM-1-mediated transmigration across the endothelium (O’Brien et al., 2003; Mocsai et al., 2015).

\section{Neutrophil Adhesion and Transendothelial Migration}

Adhesion molecules, such as selectins and integrins, work cooperatively to regulate neutrophil recruitment. Although little is known about palmitoylation of adhesion molecules expressed on the neutrophil surface, it has been seen in many cells and cell fragments that directly interact with neutrophils. For instance, in ECs, PECAM-1 is palmitoylated by DHHC21, and knockdown of DHHC21 greatly reduces the protein level of PECAM-1 and decreases its surface expression (Marin et al., 2012). Another study reports that palmitoylation of PECAM-1 at Cys595 is required for its lipid raft localization (Sardjono et al., 2006). Additionally, P-selectin in platelets mediates the formation of the platelet-neutrophil complex via binding to its ligand PSGL-1 on neutrophils, a process that aids the firm adhesion of neutrophils to activated ECs and thereby promotes leukocyte transmigration (Lisman, 2018). Recent studies have shown that P-selectin undergoes palmitoylation in platelets and that de-palmitoylation of P-selectin by APT1 greatly inhibits the expression of P-selection in platelets (Sim et al., 2007). It is plausible that this palmitoylation-regulated $\mathrm{P}$-selectin change in platelets may affect neutrophil recruitment.

Neutrophil adhesion molecules require partner protein tetraspanins for their stable membrane localization and proper function (Yeung et al., 2018). Tetraspanins are transmembrane proteins that contain a large extracellular loop to interact with adhesion molecules and other tetraspanins, forming a signal transducing complex called tetraspanin-enriched microdomains (TEMs) in cell membrane. Tetraspanins facilitate the recruitment of adhesion molecules to TEMs and amplify their intracellular signals, therefore playing important roles in regulating neutrophil function. For example, CD $37^{-/}$neutrophils exhibit an impaired ability to migrate toward chemotactic stimuli and to adhere to postcapillary venules (Wee et al., 2015); CD63 binds to Mac-1 and enhances Mac-1-mediated adhesion function (Skubitz et al., 1996). So far, a large body of tetraspanins, including CD9, CD37, CD53, CD63, CD81, CD82 and CD151, has been reported as palmitoylation substrates (Charrin et al., 2002; Yang et al., 2002; Termini et al., 2014). Investigating whether and how palmitoylation affects tetraspanin function in neutrophils is of great significance for the comprehensive understanding of the regulation of neutrophil functions.

\section{Neutrophil Chemotaxis}

Once migrated out of microvessels, neutrophils in tissues are capable of moving along chemoattractant gradients toward sites of infection or injury. Neutrophil chemotaxis starts with polarization, characterized by the formation of a leading-edge containing pseudopod and a highly contractile trailing edge (Hind et al., 2016). The migration of the neutrophil depends on dynamic polymerization and depolymerization of actin filament, which pushes the leading-edge forward and retracts the tail at the rear of the cell (Szczur et al., 2006; Mocsai et al., 2015). The key regulators of these coordinated processes are members of the small Rho GTPase family, including Rac, Cdc42, and Rho (Sun et al., 2004; Mocsai et al., 2015).

Rac is essential for neutrophil motility as well as directional sensing during migration. Similar to other members of small GTPase, Rac exists in two states: GTP-bound activated state or GDP-bound de-activated state. During neutrophil migration, GTP-bound Rac1 promotes the activation of $\mathrm{p} 21$ activated kinase (PAK) and the reorganization of actin, which subsequently aid membrane protrusion via lamellipodia formation (Filippi et al., 2007). Rac-1 is palmitoylated at Cys178, and the attachment of palmitate requires prior prenylation 


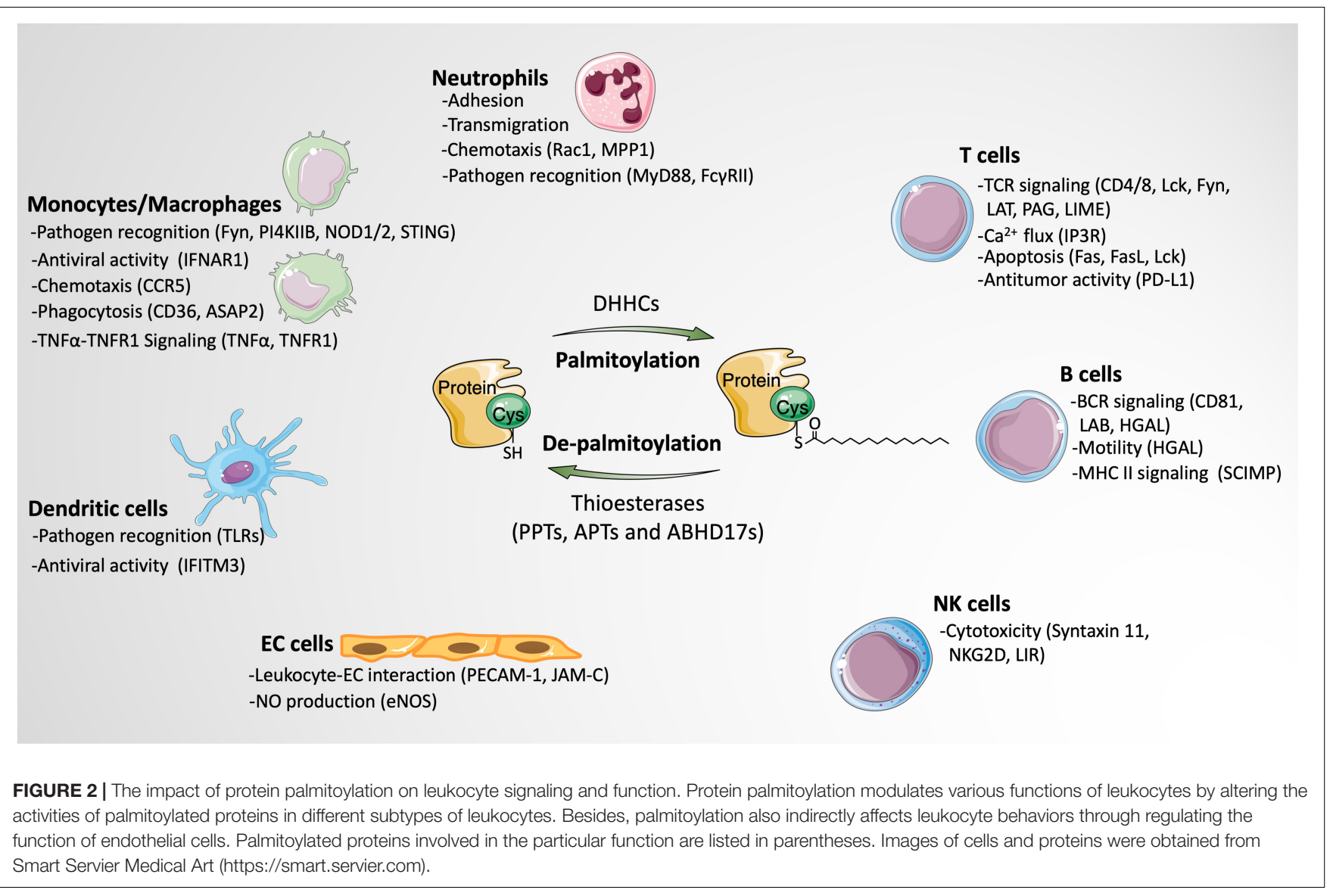

at Cys189 and a polybasic domain in C-terminal region. Palmitoylation-defective Rac1 (mutation at Cys178) shows reduced ability to load GTP and decreased lipid raft partitioning, which are accompanied by decreased PAK activity and F-actin recruitment at plasma membrane. Cells expressing Cys178 mutant Racl display defective spreading and migrate with less directionality, suggesting the essential roles of palmitoylation in regulating Racl function and neutrophil migration (NavarroLerida et al., 2012). In contrast to Rac, other members of the small GTPase family do not seem to be regulated by palmitoylation. For example, RhoA is a non-palmitoylated small GTPase (Navarro-Lerida et al., 2012), and Cdc42 only undergoes prenylation, with an exception of a brainspecific variant of Cdc42 which is palmitoylated by DHHC8 (Moutin et al., 2017).

In addition to small GTPases, other palmitoylated proteins are also involved in regulating neutrophil chemotactic migration. For example, membrane palmitoylated protein 1 (MPP1 or P55) is essential for the formation of a single pseudopod at the leading edge by confining small GTPase activation and actin polymerization to a localized region. MPP1 knockout neutrophils display multiple transient pseudopods in all directions upon stimulation and fail to perform chemotaxis efficiently (Quinn et al., 2009). However, the impacts of palmitoylation on MPP1 function remain to be addressed in neutrophils.

\section{Pathogen Recognition}

Neutrophils express several kinds of surface receptors for the recognition of invading pathogens such as Toll-like receptors (TLRs), Fc $\gamma$-receptors, and formyl-peptide receptors (Futosi et al., 2013). Growing evidence has shown that many of these receptors and their adaptor proteins are regulated by palmitoylation.

\section{Toll-like receptors/MyD88 signaling}

Toll-like receptors are responsible for recognizing pathogenassociated molecular patterns (PAMPs) and damage-associated molecular patterns (DAMPs). TLR signaling has previously been shown to be activated by saturated fatty acids even though fatty acids do not bind to TLR, suggesting a ligand-independent TLR activation meditated by fatty acids (Fessler et al., 2009; Lancaster et al., 2018). Further study reveals that MyD88, an adaptor molecule of TLR signaling, is palmitoylated by DHHC6 at Cys 113 (Kim et al., 2019). The palmitoylation of MyD88 is required for its binding to IRAK4 and activation of downstream NF- $\mathrm{kB}$. Reducing intracellular palmitate concentration by the inhibition of de novo fatty acid synthesis blocks LPS-induced neutrophil activation and MyD88-dependent cytokine production. The critical roles of palmitoylation in TLR/MyD88 signaling is further supported by the fact that the DHHC inhibitor 2-bromopalmitate (2-BP) inhibits NF- $\mathrm{kB}$ activation, whereas the APT inhibitor palmostatin B enhances the activation of NF- $\mathrm{kB}$ (Kim et al., 
2019). In fact, many isotypes of TLRs (TLR 2,5,6,10) are shown to be palmitoylated in other types of leukocytes, which will be discussed in detail later.

\section{Fc $\gamma$ receptors}

Neutrophils constitutively express Fc $\gamma$ RII (CD32) and Fc $\gamma$ RIII (CD16) while transiently expressing Fc $\gamma \mathrm{RI}$ (CD64) upon activation. Neutrophil Fc $\gamma$ receptors enable neutrophils to recognize antigens via immunoglobulins (Igs)-mediated interactions and regulate many neutrophil functions (Futosi et al., 2013; Kara et al., 2020). Thus, Fc $\gamma$ receptors serve as a crucial link between innate immune effector cells and adaptive immune responses. The function of $\mathrm{Fc} \gamma \mathrm{R}$ and its ability to bind to IgG complex requires $\mathrm{Fc} \gamma \mathrm{R}$ partitioning into lipid rafts (Bournazos et al., 2009). It has been shown that the lipid raft targeting of Fc $\gamma$ RII is regulated by palmitoylation at Cys 208 in its juxtamembrane region; mutation of Cys208 results in diminished translocation of Fc $\gamma$ RII to lipid rafts and impaired Fc $\gamma$ RII downstream signaling (Barnes et al., 2006).

The palmitoylation of surface receptors like TNFRs, Fas, and CCRs, although not yet tested in neutrophils, have been established in other immune cells (Blanpain et al., 2001; Rossin et al., 2015; Zingler et al., 2019).

\section{Monocytes/Macrophages}

Monocytes and macrophages are critical components of innate immune systems. Monocytes patrol around the circulation and eliminate pathogens via phagocytosis. Once recruited to the sites of inflammation, monocytes differentiate into macrophages or dendritic cells. In addition to phagocytosis, monocytes and macrophages also produce inflammatory mediators and function as antigen presenting cells (APCs). An ABE-coupled proteomic study reveals 98 palmitoylated proteins in macrophages, $48 \%$ of which have been reported to be localized in lipid rafts (Merrick et al., 2011). Many of the previously reported palmitoylated proteins participate in various monocyte/macrophage functions, such as syntaxin-7 in phagocytosis, SNAP-23 in TNF $\alpha$ secretion and phospholipid scramblase 3 (PLSCR3) in apoptosis (Vogel and Roche, 1999; He and Linder, 2009; Merrick et al., 2011), suggesting critical roles of palmitoylation in modulating monocyte/macrophage functions.

\section{Pathogen Recognition \\ TLR signaling pathway}

Emerging evidence demonstrates that palmitoylation is involved in LPS-induced macrophage activation. A proteomics study using RAW264 macrophage-like cells reveals that LPS stimulates global changes in protein palmitoylation, affecting nearly 340 palmitoylated proteins (Kwiatkowska and Ciesielska, 2018). More importantly, TLR signaling in macrophages is regulated by many palmitoylated proteins. Src family kinase Fyn, an inhibitory regulator of TLR4, is palmitoylated at Cys3, a modification required for its lipid raft localization and catalytic function. LPS stimulation increases the level of palmitoylated Fyn in lipid rafts where Fyn mediates the suppression of both NF- $\kappa \mathrm{B}$ and IRF3-dependent cytokine production (BorzeckaSolarz et al., 2017). Another LPS-upregulated palmitoylated protein is type II phosphatidylinositol 4-kinase (PI4KIIB). Palmitoylation of PI4KIIB triggers the activation of PI4KIIB and promotes the subsequent synthesis of $\mathrm{PI}(4,5) \mathrm{P} 2$, a phospholipid compound that can activate TLR4 signaling on its own or through its hydrolytic products DAG, IP3, and PIP3 (Sobocinska et al., 2018).

\section{Nucleotide oligomerization domain 1 (NOD1) and NOD2}

Nucleotide oligomerization domain $1 / 2$ are cytosolic pattern recognition receptors that recognize bacterial peptidoglycans and promote subsequent immune responses such as NF$\kappa \mathrm{B}$ activation and cytokine production. The interaction of NOD1/2 with membrane structures (e.g., plasma membrane and bacterial-containing phagosomes) is essential for their function. NOD1/2 are palmitoylated at multiple cysteine residues: Cys558, 567, and 952 of NOD1 and Cys395 and 1033 of NOD2 (Lu et al., 2019). Administration of 2-BP greatly inhibits the recruitment of NOD1/2 to membrane structures and the production of cytokines in macrophages treated with bacterial peptidoglycans. DHHC5 is responsible for the palmitoylation of NOD1/2; silencing or knockout of DHHC5 abrogates NOD1/2-dependent NF- $\mathrm{B}$ activation in bacterial peptidoglycans-stimulated macrophages (Lu et al., 2019).

\section{Stimulator of interferon genes (STING)}

STING recognizes cyclic dinucleotides of bacteria and DNA viruses, leading to potent cytokine production; it can also be activated by self-DNA that leaks from nucleus or mitochondria, which results in autoinflammatory diseases (Hansen et al., 2019). DHHC3, 7, and 15 mediatedpalmitoylation of STING at Cys88 and 91 is required for STING activity and the subsequent promotion of type I interferon (IFN) responses (Mukai et al., 2016). Nitrofatty acids, which directly target cysteine residues and therefore block STING palmitoylation, greatly reduce the production of type I IFN and IFN-induced CXCL10 and IL-6 in monocytes and macrophages upon virus infection (Hansen et al., 2018).

\section{Antiviral Activity}

Interferon- $\alpha / \beta$ receptor (IFNAR) is the receptor for antiviral cytokine type I IFNs that functions as a critical player in combating virus infection (Gonzalez-Navajas et al., 2012). It also possesses anti-bacterial and immunomodulatory properties by inducing the transcription of IFN-stimulated genes (Ivashkiv and Donlin, 2014). Upon binding to type I IFNs, IFNAR undergoes endocytosis and activates receptorassociated Jak kinases, which results in the phosphorylation of signal transducer and activator of transcription 1 (STAT1) and STAT2 and ultimately the transcription of the IFN-stimulated genes (Lee and Ashkar, 2018). Palmitoylation is required for the activation of IFNAR-Jak-Stat pathway. Palmitoylation inhibitor greatly diminishes the endocytosis of IFNAR1 and the phosphorylation of STAT1. Further study reveals that IFNAR1 is palmitoylated at Cys463, and mutation of Cys463 blocks the phosphorylation of STAT1 and 2 and their nuclear 
translocation, without affecting IFNAR1 endocytosis and stability (Claudinon et al., 2009).

\section{Chemotaxis}

C-C chemokine receptor type 5 (CCR5) is a chemokine receptor that belongs to the GPCR superfamily. CCR5 regulates macrophage chemotaxis and also functions as a coreceptor for the macrophage-tropic human immunodeficiency (M-tropic $\mathrm{HIV}$ ) virus entry into monocytes/macrophages and $\mathrm{CD} 4^{+} \mathrm{T}$ lymphocytes (Tuttle et al., 1998). CCR5 is palmitoylated at Cys321, 323, and 324 located between the cytoplasmic end of the seventh transmembrane domain and the C-terminal tail (Percherancier et al., 2001). Mutation of these cysteine residues leads to the greatly reduced surface expression of CCR5. Moreover, palmitoylation prevents CCR5 from degradation, thus stabilizing CCR5; palmitoylation-defective CCR5 displays a 3-fold decease in its half-life compared to WT CCR5. Functional analysis shows that palmitoylation does not affect the binding ability of CCR5 with its agonist macrophage inflammatory protein-1 $\beta$ (MIP-1 $\beta$ ). The reduced function of palmitoylation-defective CCR5 is attributed to its impaired surface expression (Blanpain et al., 2001; Percherancier et al., 2001).

\section{Phagocytosis \\ CD36}

CD36 is the primary scavenger receptor expressed on monocytes and macrophages that facilitates the phagocytosis of pathogens, dead cells, modified low-density lipoproteins, and long-chain fatty acids (Silverstein and Febbraio, 2009). Palmitoylation occurs at both N-terminal (Cys3 and Cys7) and C-terminal (Cys464 and Cys466) residues of CD36 (Meiler et al., 2013). Palmitoylation of CD36 requires ER protein Selk, the cofactor of DHHC6. Selk ${ }^{-1-}$ macrophages exhibit reduced membrane expression of $\mathrm{CD} 36$ due to the decreased level of CD36 palmitoylation (Meiler et al., 2013). It is possible that Selk tethers DHHC6 to ER membrane via its SH3-binding domain, thus promoting DHHC6-mediated palmitoylation of CD36 in ER of macrophages. However, another study of adipocyte CD36 reveals that DHHC4 and DHHC5 are actually responsible for the palmitoylation of CD36 and its stable membrane localization (Wang J. et al., 2019). In adipocytes, DHHC4-mediated palmitoylation in Golgi targets CD36 to plasma membrane, while DHHC5-mediated palmitoylation in plasma membrane prevents CD36 disassociation with plasma membrane. Whether this modification mechanism also applies to macrophages needs to be further tested.

\section{Fc $\gamma R$ signaling}

Fc $\gamma$ receptors are also expressed on macrophages, mediating the phagocytosis of IgG-coated pathogens, the secretion of inflammatory mediators, and IgG-dependent elimination of infected cells (Fitzer-Attas et al., 2000). As mentioned in section "Neutrophils- Pathogen Recognition," palmitoylation is required for Fc $\gamma \mathrm{R}$ lipid raft partitioning and its binding ability with IgG complex (Barnes et al., 2006). In addition to the receptor itself being palmitoylated, effector protein downstream of $F c \gamma R$ is also palmitoylated in macrophages. Arf-GAP with $\mathrm{SH} 3$ domain,
ANK repeat, and PH domain-containing protein 2 (ASAP2), is a scaffolding protein that regulates cytoskeletal rearrangement during Fc $\gamma$ R-mediated phagocytotic cup formation (Norton et al., 2017). Once phagocytotic cups mature, ASAP2 is cleaved from the cups by calpain-2 to allow efficient phagocytosis to occur. ASAP2 is palmitoylated at Cys86 by DHHC6/Selk in ER, and palmitoylation is required for the cleavage of ASAP2 and the subsequent maturation of the phagocytic cups. Inhibition of ASAP2 palmitoylation by mutagenesis, palmitoylation inhibitor, or Selk mutation leads to prolonged retention of ASAP2 in phagocytic cups and impaired Fc $\gamma \mathrm{R}$-mediated phagocytosis (Norton et al., 2017).

\section{TNF $\alpha$-TNFR1 Signaling}

$\mathrm{TNF} \alpha$ is a multifunctional pro-inflammatory cytokine primarily secreted by macrophages. Newly synthesized TNF $\alpha$ is a transmembrane protein $(\operatorname{tmTNF} \alpha)$. Upon stimulation, the ectodomain of tmTNF $\alpha$ is cleaved by TNF $\alpha$-converting enzyme ADAM17, producing biologically active soluble TNF $\alpha$ ( $s T N F \alpha)$. The membrane-bound $\mathrm{TNF} \alpha$ fragment is further cleaved by signal peptide peptidase-like $2 \mathrm{~b}$ (SPPL2b) to generate the intracellular domain of TNF $\alpha$ (ICD-TNF $\alpha$ ), which then activates IL- $1 \beta$ promoter. Palmitoylation of TNF $\alpha$ has been reported; the $\left[{ }^{3} \mathrm{H}\right]$ labeled palmitate is detected in tmTNF $\alpha$ but not in sTNFa (Utsumi et al., 2001). Mutation of the palmitoylation site of tmTNF $\alpha$ does not affect ADAM17dependent cleavage of its ectodomain. However, palmitoylation of tmTNF $\alpha$ regulates the sensitivity of $\mathrm{TNF} \alpha$ receptor 1 (TNFR1). Palmitoylation facilitates tmTNF $\alpha$ partitioning to the lipid rafts, where it suppresses the activity of TNFR1 by competing with sTNF $\alpha$. Whereas, palmitoylation-defective tmTNF $\alpha$ shows reduced ability to block the binding of sTNF $\alpha$ to TNFR1 and the transcription of TNF $\alpha$ target genes (Poggi et al., 2013). Palmitoylation is also required for the enzymatic cleavage of the membrane-bound TNF $\alpha$ fragment by SPPL2b. Mutagenesis of the palmitoylation site of TNF $\alpha$ results in a rapid degradation of the membrane-bound TNF $\alpha$ fragment prior to SPPL2b-mediated cleavage, thus reducing ICDTNF $\alpha$ production and downstream IL- $1 \beta$ promoter activation (Poggi et al., 2013).

The majority of TNF $\alpha$ biological function is mediated by TNFR1, which belongs to a subgroup of death receptors. Upon binding to TNF $\alpha$, TNFR1 induces two distinctive downstream signaling pathways depending on its subcellular localization. TNFR1 in plasma membrane signals for NF$\kappa \mathrm{B}$ activation, while internalized TNFR1 activates apoptosis signaling. TNFR1 is palmitoylated at Cys248, which is required for its plasma membrane localization in human monocytic U937 cells (Zingler et al., 2019). Mutagenesis of Cys248 results in decreased TNFR1 expression in plasma membrane, reducing both NF- $\mathrm{KB}$ and apoptosis signaling. Furthermore, activated TNFR1 undergoes APT2-mediated de-palmitoylation, which promotes TNFR1 translocation to designated signaling platforms to activate NF- $\mathrm{\kappa B}$. APT2 inhibitor blocks TNFR1 de-palmitoylation and its downstream NF- $\mathrm{KB}$ activation but enhances TNFR1-mediated apoptosis signaling (Zingler et al., 2019). 


\section{Dendritic Cells}

Dendritic cells (DCs), functioning as the most potent APCs, are specialized to recognize, phagocytize, process, and present antigens to both memory and naïve $T$ cells (Steinman and Banchereau, 2007). There is limited information regarding the roles of palmitoylation in DCs. Two Alk-C16 metabolic labeling-based palmitoyl proteomic studies reveal hundreds of palmitoylated proteins with diverse cellular function in DCs, such as TLR2 for pathogen recognition; IFITM3 for antiviral activity; G protein subunits, N-Ras and Lyn for signal transduction; CD81 for DC migration; and CD80 and 86 for immunomodulation (Yount et al., 2010; Quast et al., 2011; Chesarino et al., 2014; Yeung et al., 2018). Among these proteins, only TLR2 and IFITM3 palmitoylation have been investigated for their functional impact in DCs.

\section{Pathogen Recognition Toll-like receptors}

In DCs, TLR2 is found to be palmitoylated on Cys609 at the cytoplasmic edge of the transmembrane domain (Chesarino et al., 2014). Multiple DHHCs (DHHC 2, 3, 6, 7, 15) are responsible for the palmitoylation of TLR2. Inhibition of TLR2 palmitoylation either by 2-BP or mutation of the palmitoylation site leads to the decreased surface expression of TLR2 in DCs, without affecting the overall protein level and stability of TLR2. Blocking TLR2 palmitoylation also inhibits microbial ligands-induced activation of NF-KB and production of IL- 6 and TNF $\alpha$, which is, at least partially, due to the insufficient surface expression of TLR2. Similar to TLR2, other TLRs (TLR5,6,10) have been shown to be palmitoylated in DCs (Chesarino et al., 2014). Whether and how palmitoylation regulates their function remain to be addressed.

\section{Antiviral Activity}

Interferon-induced transmembrane protein 3 (IFITM3), an INFstimulated effector protein, plays important roles in restricting virus infection in host cells. Upon virus infection, IFITM3 interacts with the incoming virus particles and directs them to lysosomes for elimination. The essential roles of palmitoylation in the antiviral activity of IFITM3 have been well-recognized (Yount et al., 2010; Spence et al., 2019). In DCs, IFITM3 is palmitoylated on Cys71, Cys72 and Cys105 in the transmembrane proximal region (Yount et al., 2010). Mutation of IFITM3 palmitoylation sites does not affect the expression or subcellular localization of IFITM3 in DCs at steady state; however, palmitoylation-defective IFITM3 exhibits a greatly reduced ability to cluster with virus particles and impaired antivirus activity (Yount et al., 2010; Spence et al., 2019).

\section{T Cells}

$\mathrm{T}$ cells coordinate a variety of adaptive immune responses. The pivotal roles of protein palmitoylation in regulating $\mathrm{T}$ cell functions have been well recognized.

\section{T Cell Receptor Signaling}

$\mathrm{T}$ cells are activated by the binding of TCRs to the major histocompatibility complex (MHC):antigen peptide complex. Briefly, the TCR-pMHC engagement facilitated by co-receptors
CD4/CD8 activates tyrosine kinase Lck and Fyn to phosphorylate the TCR/CD3 $\zeta$-chain, recruiting tyrosine kinase ZAP-70 to phosphorylate transmembrane adaptor proteins (LAT, PAG, etc.). This initiates the propagation of several signaling pathways and eventually leads to the activation of the transcription factors NFAT, NF- $\mathrm{B}$, and AP-1. One study has shown that 2-BP disrupts signaling protein localization and blocks TCR signaling as evidenced by reduced tyrosine phosphorylation and impaired calcium influx (Webb et al., 2000). Moreover, several palmitoyl proteomic studies reveal that although T-cell receptor itself does not undergo palmitoylation, TCR co-receptors CD4, CD8, Src family kinases Lck and Fyn, and adaptor proteins LAT and PAG are all palmitoylated (Martin and Cravatt, 2009; Morrison et al., 2015). How palmitoylation regulates the function of TCR signaling proteins has been previously reviewed (Bijlmakers, 2009; Ladygina et al., 2011); therefore, it will only be briefly discussed in this section. Signaling effectors mentioned in previous sections like Ras and Rac will not be covered again in this section.

\section{$T$ cell receptor coreceptors $C D 4 / C D 8$}

The extracellular domain of CD4 and CD8 binds to the nonpolymorphic regions of Class II and Class I MHC on APCs, respectively, facilitating the interaction of TCR with pMHC. Meanwhile, their intracellular cytoplasmic tails are closely associated with Lck (Artyomov et al., 2010; Courtney et al., 2018). Palmitoylation of CD4 occurs at Cys394 and Cys397 residues adjacent to the transmembrane domain (Crise and Rose, 1992). Palmitoylation functions as the lipid raft targeting signal of $\mathrm{CD} 4$, facilitating its partitioning in lipid raft and clustering with TCR and PKC $\theta$ there (Balamuth et al., 2004). CD4 also serves as a key coreceptor for HIV entry in T cells, and current research indicates that CD4 palmitoylation is not required for this function (Bijlmakers, 2009). CD8 is heterodimer consisting of $\mathrm{CD} 8 \alpha$ and $\mathrm{CD} 8 \beta$. Species-specific palmitoylation of $\mathrm{CD} 8$ has been reported. In mice, only $\mathrm{CD} 8 \beta$, but not $\mathrm{CD} 8 \alpha$, undergoes palmitoylation on Cys179 in its cytoplasmic domain (Arcaro et al., 2001). In contrast, human CD8 $\beta$ contains two palmitoylated cysteine residues, and human CD8 $\alpha$ is also palmitoylated. In mouse T cells, mutation of Cys179 abolishes CD8 lipid raft localization and impairs its association with Lck (Arcaro et al., 2001). Unexpectedly, palmitoylation of human CD8 does not seem to play a role in targeting CD8 to lipid raft (Pang et al., 2007). Instead, lipid raft targeting of CD8 solely relies on the positively charged amino acids in CD8 $\beta$ (Bijlmakers, 2009). The impact of palmitoylation on other functions of CD8 still remains unclear.

\section{Src family kinases}

Lck is actively involved in TCR signaling as well as T cell development (Molina et al., 1992; Palacios and Weiss, 2004). Lck is a dually acylated protein (Resh, 1994). Upon synthesis, Lck is myristoylated at Gly2, which is required for the subsequent palmitoylation at Cys3 and Cys5. Stable association between Lck and plasma membrane, which is critical for the activity of Lck, is mediated by palmitoylation at both Cys3 and Cys5 (Yurchak and Sefton, 1995). Multiple DHHCs (2, 3, 17, 18, 21) 
are responsible for the palmitoylation of Lck (Aicart-Ramos et al., 2011; Zeidman et al., 2011; Akimzhanov and Boehning, 2015). Unexpectedly, Lck is mainly localized outside of lipid rafts in steady state $\mathrm{T}$ cells, yet $\mathrm{T}$ cell activation accelerates the palmitate turnover of Lck, which promotes the translocation of Lck into lipid rafts to phosphorylate Fyn and other substrates (Kosugi et al., 2001). Lck with mutation at both palmitoylation sites appears in a soluble form and is unable to interact with CD4 and promote downstream signaling events (Kabouridis et al., 1997). Fyn is another highly expressed Src family kinase in T cells. The absence of Fyn leads to reduced responses to TCR stimulation suggesting that Fyn is also involved in TCR signaling (Rodolfo et al., 1991; Tsygankov et al., 1996; Yasuda et al., 2002). Similar to Lck, Fyn also undergoes both myristoylation and palmitoylation. Attachment of palmitate can occur on both Cys3 and Cys6, although the majority of Fyn is only palmitoylated on Cys3 (Liang et al., 2001). The attached palmitate on Cys3 (with a half-life of 1.5-2 h) is important for membrane association and lipid raft sequestering of Fyn (Wolven et al., 1997). DHHC2,3,7,10,15,20 and 21 have all been shown to catalyze the palmitoylation of Fyn (Mill et al., 2009). In resting T cells, Fyn, unlike Lck, mainly resides in lipid rafts. Upon TCR ligation, Fyn is activated by Lck in lipid rafts which, in turn, catalyzes the phosphorylation of the TCR/CD3 $\zeta$-chain and transduces the signals to downstream effectors (Filipp et al., 2003).

\section{Transmembrane adaptor proteins (TRAPs)}

TRAPs serve as the functional connection between TCR complex and intracellular signaling molecules. Following TCR ligation, TRAPs are phosphorylated by activated Src family kinases and ZAP-70, allowing the subsequent recruitment of cytoplasmic signaling effectors and the propagation of TCR-elicited signals into cytoplasm (Horejsi et al., 2004). Among all TRAPs, LAT is indispensable for peripheral $\mathrm{T}$ cell activation due to its ability to recruit and activate many key signaling molecules including PLC $\gamma 1$, PI3K, Grb2, Grads, and SLP76 (Horejsi, 2004). LAT is palmitoylated at Cys26 and Cys29 in the juxtamembrane Cys$\mathrm{X}$-X-Cys motif. LAT palmitoylation is essential for the stability of LAT and its association with plasma membranes; mutation in two palmitoylation sites results in a remarkably reduced LAT expression and the retention of LAT in Golgi complex (Shogomori et al., 2005; Tanimura et al., 2006). Moreover, palmitoylation-defective LAT is not capable of interacting with TCR complex or recruiting PLC $\gamma$ and Grb2 to propagate signals (Harder and Kuhn, 2000). T cells expressing palmitoylationdefective LAT display impaired $\mathrm{Ca}^{2+}$ influx, ERK activation, and transcription factor induction upon $\mathrm{T}$ cell activation (Zhang et al., 1999). Furthermore, LAT palmitoylation is closely related to $\mathrm{T}$ cell anergy. Compared to control $\mathrm{T}$ cells, anergic $\mathrm{T}$ cells display profoundly reduced LAT palmitoylation, which leads to reduced LAT recruitment to immunological synapses and lipid rafts (Hundt et al., 2006). Functionally, LAT in anergic T cells shows a reduced phosphorylation level upon TCR stimulation and an impaired ability to activate downstream PLC $\gamma$ and PI3K (Hundt et al., 2006; Ladygina et al., 2011).

Other TRAPs like phosphoprotein associated with GEMs (PAG) and Lck-interacting membrane protein (LIME) are also palmitoylated at the same Cys-X-X-Cys motif in T cells, and both are concentrated in lipid rafts (Ladygina et al., 2011). $\mathrm{PAG}$, also known as Csk-binding protein (Cbp), acts as negative regulator of T-cell signaling. PAG is capable of recruiting Csk to inhibit Src family kinases via phosphorylating tyrosine residues in their C-terminal regulatory domains. Upon TCR ligation, PAG rapidly releases Csk, resulting in the activation of Lck and Fyn (Davidson et al., 2003). PAG with a mutation in its palmitoylation motif displays normal expression level and plasma membrane localization, but it no longer resides in lipid rafts. Although it is able to recruit Csk, palmitoylation-defective PAG cannot inhibit proximal TCR signaling (Posevitz-Fejfar et al., 2008). Similar to PAG, LIME can also bind to Csk and Lck, yet the biological function of LIME in T cells remains unclear. One study has shown that LIME contributes to the activation of ERK and JNK and the production of IL-2 in Jurkat T cells (Hur et al., 2003). The attached palmitates at Cys 28 and Cys 31 in the Cys-X-X-Cys motif act as lipid raft targeting signals for LIME (Hur et al., 2003). However, the functional significance of palmitoylation on LIME has not yet been investigated.

\section{Calcium Flux}

Upon TCR activation, intracellular signaling effector PLC $\gamma$ hydrolyzes PI $(4,5) \mathrm{P} 2$ to produce DAG and IP3; IP3, in turn, triggers $\mathrm{Ca}^{2+}$ release from ER stores via binding to IP3Rs on ER membrane (Nagaleekar et al., 2008). IP3Rs are palmitoylated by DHHC6/Selk complex in ER. The putative palmitoylation sites are reported to be at Cys56, 849, and 2214, which needs further confirmation (Fredericks et al., 2014). Knockdown of DHHC6 significantly reduces the expression of IP3R on ER membrane and attenuates IP3R-dependent $\mathrm{Ca}^{2+}$ flux in Jurkat $\mathrm{T}$ cells (Fredericks et al., 2014). The proper function of DHHC6 requires binding to its cofactor Selk via the SH3-binding domain in Selk. Selk deficiency also leads to decreased IP3R level, probably due to increased IP3R proteolysis. In fact, this DHHC/Selk-dependent regulation of $\mathrm{Ca}^{2+}$ release is shared by many immune cells. In Selk knockout mice, receptor-mediated $\mathrm{Ca}^{2+}$ flux is impaired in $\mathrm{T}$ cells, neutrophils, and macrophages, resulting in disrupted $\mathrm{T}$ cell proliferation, neutrophil migration, and macrophage oxidative burst (Verma et al., 2011).

\section{Apoptosis}

The ligation of Fas and FasL induces apoptosis of $\mathrm{T}$ cells that recognize self-antigens or are activated repeatedly, key for eliminating autoreactive $\mathrm{T}$ cells and preventing autoimmune diseases (Volpe et al., 2016). Palmitoylation plays critical roles in regulating the Fas-FasL signaling pathway in T cells as both Fas and FasL are palmitoylation substrates. Fas (also known as CD95, or APO1 or TNFRSF6) is a type I transmembrane protein that contains a death domain in its cytoplasmic region. Upon binding to FasL, Fas recruits death-inducing signaling complex (DISC), which, in turn, activates caspase- 3 and induces programmed cell death. Promoting apoptosis signals requires Fas partition into actin cytoskeleton-linked lipid rafts and then internalization, both of which are regulated by Fas palmitoylation (Rossin et al., 2015). Mouse Fas is palmitoylated at Cys 194 adjacent to the transmembrane domain, while human Fas is palmitoylated at 
Cys199. One study shows that DHHC7 is responsible for the palmitoylation of Fas, and this modification stabilizes Fas by preventing its degradation by lysosomes (Rossin et al., 2015). Palmitoylation-defective Fas exerts severely reduced lipid raft localization, which impairs DISC assembly, Fas internalization, and T cell apoptosis (Chakrabandhu et al., 2007; Cruz et al., 2016). The impaired apoptosis-inducing ability of palmitoylationdefective Fas can also be observed in other Fas-expressing immune cells, like B cells and dendritic cells (Cruz et al., 2016).

Palmitoylation also participates in the signaling propagation downstream of Fas ligation, including Lck activation, PLC- $\gamma 1$ phosphorylation, and proapoptotic $\mathrm{Ca}^{2+}$ release. 17-ODYA metabolic labeling reveals that Fas ligation leads to a rapid increase in Lck palmitoylation followed by quick depalmitoylation, catalyzed by DHHC21 and APT1, respectively (Akimzhanov and Boehning, 2015). This rapid dynamic of Lck palmitoylation matches the activation kinetics of other signaling molecules subsequent to Fas stimulation. Re-expressing palmitoylation-defective Lck is unable to induce Fas-mediated $\mathrm{Ca}^{2+}$ release, caspase- 3 activation, or cell death in Lck-deficient Jurkat T cells (Akimzhanov and Boehning, 2015). It is worth noting that, in abnormally or excessively activated $\mathrm{T}$ cells, the membrane FasL can be cleaved by metalloproteases (e.g., ADAM10 and AMDA17) to generate sFasL. sFasL binding to Fas is unable to activate Fas, thus sFasL serves as a competitor of FasL to suppress FasL-induced apoptosis (Suda et al., 1997). Shedding of FasL requires the colocalization of FasL, metalloproteinases, and Lck in lipid rafts. The lipid raft targeting of both FasL and Lck depends on palmitoylation, with FasL being palmitoylated on Cys82 and Lck at Cys3 and Cys5. Inhibition of palmitoylation with 2-BP remarkably blocks FasL shedding in $\mathrm{T}$ cells (Ebsen et al., 2015).

\section{Antitumor Activity}

Programmed cell death 1 (PD1), an inhibitory surface receptor of $\mathrm{T}$ cells, suppresses $\mathrm{T}$ cell activation through binding to its ligands programmed-death ligand 1 (PD-L1) and PD-L2, playing essential roles in self-tolerance. Many tumor cells often express high level of PD-L1, which inhibits the antitumor responses of T cells such as cytokine production and cytotoxicity (Juneja et al., 2017). PD-L1 is palmitoylated by DHHC3 in its cytoplasmic domain, and the attachment of palmitic acid stabilizes PD-L1 due to decreased ubiquitination. Inhibiting PD-L1 palmitoylation via 2-BP or silencing of DHHC3 greatly reduces the expression of PD-L1 in tumor cells and therefore promotes $\mathrm{T}$ cell-mediated antitumor immunity (Yao et al., 2019).

\section{B Cells}

B cells are specialized in producing highly specific antibodies in response to diverse foreign antigens, thus playing critical roles in adaptive immunity. They can also serve as APCs and produce a variety of cytokines (IL-6, IL-10, CXCL13, and CCL19, etc.), influencing the function of other immune cells (Fillatreau, 2018). Palmitoylation has been studied less in B cells compared to $\mathrm{T}$ cells. An ABE-based palmitoyl proteomic study in human B cells reveals over 100 palmitoylated proteins or candidates that are known to participate in diverse cellular events of B cells (e.g., signal transduction, vesicle fusion, molecular transport, metabolism and immune responses), highlighting the functional significance of palmitoylation in regulating $\mathrm{B}$ cell behaviors (Ivaldi et al., 2012).

\section{B Cell Receptor Signaling $B$ cell receptor $(B C R)$ coreceptors}

$B$ cell receptors binding to complement-tagged antigens requires the BCR coreceptor complex CD19/CD21/CD81 as they significantly lower the threshold of BCR activation to antigens. Upon BCR ligation, the BCR-CD19/CD21/CD81 complex translocates to lipid rafts for the efficient propagation of BCRelicited signals. Similar to TCR, none of the BCR subunits are palmitoylated; yet, its co-receptor CD81 is a well-established palmitoylation substrate. CD81, which belongs to the tetraspanin superfamily, is palmitoylated at six cysteine residues in its juxtamembrane domain (Delandre et al., 2009; Yeung et al., 2018). The association of BCR and CD19/CD21/CD81 following BCR ligation rapidly promotes the palmitoylation of CD81, which regulates BCR signaling at many levels (Flaumenhaft and Sim, 2005). First, the prolonged residency of BCRCD19/CD21/CD81 in lipid rafts depends on the palmitates attached to CD81 (Cherukuri et al., 2004). Moreover, the binding of CD81 to other co-receptors requires palmitoylation. Palmitoylation-defective CD81 exhibits impaired association with CD19 (Delandre et al., 2009). Finally, palmitoylation of CD81 affects the ability of CD81 to recruit the downstream signaling molecule $14-3-3$, an adaptor protein regulating the activities of PKCs and PI3K (van Hemert et al., 2001). Inhibition of palmitoylation by 2-BP blocks BCR signaling activation, as evidenced by reduced PLC $\gamma 2$ phosphorylation and $\mathrm{Ca}^{2+}$ mobilization (Cherukuri et al., 2004). Interestingly, CD81 palmitoylation is significantly decreased in the presence of the oxidative stress, indicating CD81 may be responsible for redox stress-induced B cell responses (Clark et al., 2004).

\section{Adaptor proteins}

Linker for activation of B cells (LAB), also known as non$\mathrm{T}$ cell activation linker (NTAL), is a member of TRAPs that regulates BCR signaling. Similar to LAT, LAB is palmitoylated in a cytoplasmic juxtamembrane Cys-X-X-Cys motif and is therefore enriched in lipid rafts (Lupica et al., 1990; Brdicka et al., 2002). However, the functional impacts of LAB palmitoylation have not been explored.

\section{Motility}

Human germinal center-associated lymphoma (HGAL) is a newly identified adaptor protein regulating BCR signaling and B cell motility. HGAL is located in both plasma membrane and cytoplasm. Plasma membrane HGAL enhances BCR signaling by increasing Syk activity and its downstream $\mathrm{Ca}^{2+}$ mobilization (Romero-Camarero et al., 2013); cytosolic HGAL inhibits spontaneous and chemoattractant-induced B cell motility via suppressing the interaction between myosin and actin. The shuttling of HGAL between the cytoplasm to the plasma membrane requires the acylation modification as HGAL does not contain transmembrane domains. $\left[{ }^{3} \mathrm{H}\right]$ palmitate labeling reveals that HGAL is palmitoylated at the Cys43-F-Cys45 motif 
(Lu et al., 2015). Upon BCR ligation, palmitoylation, together with myristoylation at Gly2, facilitate the translocation of HGAL into lipid rafts where it binds to Syk and enhances Syk activity. HGAL with mutation at both palmitoylation sites fails to promote the phosphorylation of Syk and downstream $\mathrm{Ca}^{2+}$ influx in the presence of BCR stimulation. However, acylation site mutation of HGAL results in the accumulation of HGAL in cytoplasm and therefore induces a further reduction in $\mathrm{B}$ cell motility in response to chemoattractants (Lu et al., 2015).

\section{MHC II Signaling}

SLP65/SLP76, Csk-interacting membrane protein (SCIMP) belongs to the TRAP family and is involved in MHC II signaling in B cells during antigen presentation. SCIMP is mainly localized in lipid rafts and directly binds to Lyn. Upon MHC II stimulation, SCIMP is phosphorylated by Lyn, which, in turn, serves as a recruiting platform to interact with SLP65/SLP76, Csk, CD81, and Grb2 and regulates downstream MHC II signals. SCIMP is palmitoylated at its juxtamembrane palmitoylation motif (CysX-Cys; Draber et al., 2011. The roles of palmitoylation in SCIMP function, however, remain unclear.

Other palmitoylated proteins have been identified and confirmed in B cells (Ivaldi et al., 2012). For example, CD20, a four-transmembrane protein that participates in $B$ cell development and differentiation, is palmitoylated on Cys111 and Cys220 in its intracellular domain; the low-affinity IgE receptor FceRII (CD23) is a transmembrane glycoprotein involved in the regulation of IgE synthesis. Palmitoylation can occur at both Cys17 and Cys18 within the intracellular domain of CD23 (Ivaldi et al., 2012). The functional impacts of palmitoylation on these proteins have not yet been investigated. Since B cells share several common signaling pathways with other immune cells, many of the aforementioned palmitoylated proteins like Ras, G $\alpha$, Lck and Fyn, IP3R, and surface receptors will not be discussed again in this section.

\section{Natural Killer Cells}

Natural killer cells are potent cytotoxic lymphocytes that mediate the elimination of potentially harmful cells such as pathogeninfected cells, tumor cells, and cells from transplanted organs. The cytotoxicity is tightly controlled by activating receptors (e.g., NKG2D) that recognize cellular stress ligands and by inhibitory receptors (e.g., LIR and KIR) that recognize MHC class I proteins on target cells (Pegram et al., 2011). So far, there are only a few studies demonstrating that palmitoylation regulates $\mathrm{NK}$ cytotoxicity, by either modulating secretory lysosome exocytosis in NK cells or affecting NK receptor ligands in target cells.

\section{Syntaxin 11}

Upon ligation of activating receptors, NK cells, together with its target cells, form immunological synapses at contact points. NK cells undergo secretory lysosome exocytosis, during which the secretory lysosomes move toward immunological synapses and fuse with plasma membrane to release their cytotoxic contents (e.g., perforin and granzymes). Syntaxin 11, a soluble $\mathrm{N}$-ethylmaleimide-sensitive factor attachment protein receptor (SNARE), catalyzes the membrane fusion reaction via its interaction with trans-SNARE in the opposing membrane. Syntaxin 11 is palmitoylated in its C-terminal cysteine-enriched domain in NK cells (Hellewell et al., 2014). The attached palmitate is required for the association of syntaxin 11 with cell membrane as syntaxin 11 does not contain a transmembrane domain. Mutation of cysteine residues in syntaxin 11 leads to the retention of syntaxin 11 in cytoplasm, causing impaired secretory lysosome exocytosis and reduced NK cytotoxicity (Hellewell et al., 2014).

\section{Natural killer group 2 member D (NKG2D)}

NKG2D is an activating receptor abundantly expressed on NK cells, mediating the cytotoxicity of NK cells (Wensveen et al., 2018). NKG2D itself has not been reported to be palmitoylated; however, its ligand MHC-class-I-related chain A (MICA) undergoes palmitoylation modification at Cys306 and Cys307 in its cytoplasmic tail (Aguera-Gonzalez et al., 2011). The majority of MICA is expressed on cell membranes of stressed target cells, yet MICA can also be clustered into lipid rafts to be cleaved by metalloproteases to produce soluble MICA, a negative regulator of NKG2D expression and activation. Palmitoylation is required for MICA translocation to lipid rafts and its subsequent shedding; mutation of palmitoylation sites greatly reduces the production of soluble MICA (Aguera-Gonzalez et al., 2011).

\section{Leukocyte ig-like receptor (LIR)}

LIR is an inhibitor receptor on NK cells that binds to MHC class I proteins on target cells, suppressing NK cell cytotoxicity and preventing NK cells from lysing normal "self" cells. MHC class I proteins are glycoproteins expressed on the plasma membrane of all nucleated cells in vertebrates (Hewitt, 2003). Some of the MHC class I proteins share the same unique cysteine residues in their cytoplasmic tails. Mutation of these cysteine residues abolishes the palmitoylation modification on these proteins. Concomitantly, their surface expression, extracellular confirmation, and interaction with LIR are all disrupted by cysteine residue mutation (Gruda et al., 2007). Whether palmitoylation plays a causative role in these impairments needs to be further elucidated.

\section{INDIRECT REGULATION OF LEUKOCYTE FUNCTION BY EC PROTEIN PALMITOYLATION}

Palmitoylation also regulates the function of non-immune cells that interact with leukocytes, thus indirectly affecting leukocyte functions and behaviors. A key initiation event of inflammation is the engagement of ECs with leukocytes. Our previous observations with ECs indicate that inflammatory mediators trigger a rapid increase in endothelial palmitoylated proteins (Beard et al., 2016). Palmitoylation is involved in many endothelial responses, such as the surface expression of adhesion molecules, secretion of vasoactive molecules (e.g., nitric oxide), and EC junction integrity. In our previous study, we show that the palmitoylation inhibitor 2-BP greatly attenuates the ICAM-1 surface expression of ECs in response to IL-1 $\beta$ 
and blocks leukocyte-EC adhesion in vitro. In rats subjected to LPS stimulation, leukocyte slow-rolling and adhesion to postcapillary venules are remarkably inhibited by 2-BP. DHHC21 serves as one responsible PAT mediating leukocyte adhesion since DHHC21 loss-of-function greatly reduces the number of slow-rolling and adherent leukocytes in LPS-treated mice (Beard et al., 2016). In addition, junctional adhesion molecule c (JAM-C) is another palmitoylation-modified adhesion molecule that regulates leukocyte behaviors. JAM-C belongs to the immunoglobin superfamily and contains the PDZ-binding motif. Endothelial JAM-C is located at cell-cell contact, especially in tight junctions, interacting with other PDZ motif-containing molecules like JAM-B and ZO-1. JAM-C and JAM-B promote leukocyte adhesion and transmigration due to their ability to act as counterreceptors for Mac-1 and VLA-4, respectively (Bradfield et al., 2007; Scheiermann et al., 2009). JAM-C is palmitoylated at Cys264 and 265 by DHHC7, and the palmitoylation of JAM-C is required for its tight junction localization. Functionally, palmitoylation of JAM-C affects the transmigration of lung cancer cells (Aramsangtienchai et al., 2017). However, the functional impacts of JAM-C palmitoylation on leukocyte adhesion and transmigration need to be further investigated.

Endothelial nitric oxide synthase, which produces nitric oxide (NO), is a well-established palmitoylated substrate. Beyond its vasodilation function, eNOS-derived NO also exhibits important anti-inflammatory effects. Many studies have established that NO suppresses leukocyte adhesion, partly due to its ability to decrease the expression of adhesion molecules both on ECs and leukocytes, like VCAM-1, MCP-1 and CD11/CD18 (Shu et al., 2015). eNOS is primarily localized in Golgi and caveolae (Liu et al., 1996). It is dually acylated by both N-myristoylation at Gly2 and palmitoylation at Cys15 and Cys26 (Sessa et al., 1993; Liu et al., 1995). Palmitoylation of eNOS in ECs is highly dynamic with a turnover rate of $45 \mathrm{~min}$. Multiple DHHCs $(2,3,7,8,21)$ participate in the palmitoylation of eNOS. Inhibiting palmitoylation of eNOS via knockdown of DHHC21 leads to eNOS mislocalization and impaired production of NO in ECs (Liu et al., 1995; Fernandez-Hernando et al., 2006). It is plausible that this reduction in NO caused by DHHC21 knockdown then alters leukocyte function and behaviors.

\section{CONCLUDING REMARKS AND PERSPECTIVE}

The dynamic reaction of palmitoylation and de-palmitoylation rapidly and effectively regulates protein conformation, localization, lipid raft partitioning, intracellular trafficking, stability, and interactions with other proteins in leukocytes.

\section{REFERENCES}

Aguera-Gonzalez, S., Gross, C. C., Fernandez-Messina, L., Ashiru, O., Esteso, G., Hang, H. C., et al. (2011). Palmitoylation of MICA, a ligand for NKG2D, mediates its recruitment to membrane microdomains and promotes its shedding. Eur. J. Immunol. 41, 3667-3676. doi: 10.1002/eji.201141645
Protein palmitoylation has great impact on many aspects of leukocyte function, including adhesion, transmigration, chemotaxis, phagocytosis, pathogen recognition, TCR and BCR signaling activation, cytotoxicity, cytokine production, and apoptosis. Despite the advancement in the field of palmitoylation, many critical issues remain. First, hundreds of putative palmitoylated proteins have been identified in leukocytes via large-scale palmitoyl proteomics, yet only a very small portion of them have been validated for functional impact. The large body of unexplored data contains extensive novel knowledge on palmitoylation in leukocytes that requires further investigation. Second, current understanding on how palmitoylation affects leukocyte proteins has been primarily concentrated on palmitoylation-mediated protein partitioning into membrane and/or lipid rafts; yet other regulatory mechanisms of palmitoylation have been studied to a much lesser extent. To acquire more comprehensive knowledge on palmitoylation, it will be essential to also understand its relationship with other PTMs (e.g., phosphorylation and ubiquitination), its impact on protein conformation, and its ability to mediate protein-protein interaction. Finally, our current knowledge on enzymes that catalyze palmitoylation and de-palmitoylation is still very limited, and the molecular basis of substrate recognition remains largely unknown. There is an urgent need for developing DHHC-selective inhibitors, as the most commonly used inhibitor 2-BP has been shown to exert off-target effects by interfering with fatty acid metabolism (Davda et al., 2013). Mechanisms regulating DHHC activity and substrate specificity will serve as a key in the development of DHHC specific inhibitors.

Taken together, palmitoylation influences many aspects of leukocyte function by altering protein localization and activity. Targeting palmitoylation may serve as a promising therapeutic strategy for aberrant immune responses during inflammatory injury, immune disorders, and infectious diseases.

\section{AUTHOR CONTRIBUTIONS}

XY performed literature search, drafted the manuscript, and prepared the figures. YM, VC, and SYY edited and revised the manuscript. EZ edited the manuscript and revised the figures. SYY initiated, directed, and sponsored the work throughout all levels of development. All authors approved it for publication.

\section{FUNDING}

This work was supported by NIH R35 HL150732 (to SYY) and R01 GM097270 (to SYY). 
Alonso, M. A., and Millan, J. (2001). The role of lipid rafts in signalling and membrane trafficking in T lymphocytes. J. Cell Sci. 114(Pt 22), 3957-3965.

Aramsangtienchai, P., Spiegelman, N. A., Cao, J., and Lin, H. (2017). SPalmitoylation of Junctional Adhesion Molecule C Regulates Its Tight Junction Localization and Cell Migration. J. Biol. Chem. 292, 5325-5334. doi: 10.1074/ jbc.M116.730523

Arcaro, A., Gregoire, C., Bakker, T. R., Baldi, L., Jordan, M., Goffin, L., et al. (2001). CD8beta endows CD8 with efficient coreceptor function by coupling $\mathrm{T}$ cell receptor/CD3 to raft-associated CD8/p56(lck) complexes. J. Exp. Med. 194, 1485-1495. doi: 10.1084/jem.194.10.1485

Artyomov, M. N., Lis, M., Devadas, S., Davis, M. M., and Chakraborty, A. K. (2010). CD4 and CD8 binding to MHC molecules primarily acts to enhance Lck delivery. Proc. Natl. Acad. Sci. U S A 107, 16916-16921. doi: 10.1073/pnas. 1010568107

Baker, T. L., Zheng, H., Walker, J., Coloff, J. L., and Buss, J. E. (2003). Distinct rates of palmitate turnover on membrane-bound cellular and oncogenic $\mathrm{H}$-ras. J. Biol. Chem. 278, 19292-19300. doi: 10.1074/jbc.M206956200

Balamuth, F., Brogdon, J. L., and Bottomly, K. (2004). CD4 raft association and signaling regulate molecular clustering at the immunological synapse site. J. Immunol. 172, 5887-5892. doi: 10.4049/jimmunol.172.10.5887

Barnes, N. C., Powell, M. S., Trist, H. M., Gavin, A. L., Wines, B. D., and Hogarth, P. M. (2006). Raft localisation of FcgammaRIIa and efficient signaling are dependent on palmitoylation of cysteine 208. Immunol. Lett. 104, 118-123. doi: 10.1016/j.imlet.2005.11.007

Baumgart, F., Corral-Escariz, M., Perez-Gil, J., and Rodriguez-Crespo, I. (2010). Palmitoylation of R-Ras by human DHHC19, a palmitoyl transferase with a CaaX box. Biochim. Biophys. Acta 1798, 592-604. doi: 10.1016/j.bbamem.2010. 01.002

Beard, R. S. Jr., Yang, X., Meegan, J. E., Overstreet, J. W., and Yang, C. G. (2016). Palmitoyl acyltransferase DHHC21 mediates endothelial dysfunction in systemic inflammatory response syndrome. Nat. Commun. 7:12823. doi: $10.1038 /$ ncomms 12823

Berg, V., Rusch, M., Vartak, N., Jungst, C., Schauss, A., Waldmann, H., et al. (2015). miRs-138 and -424 control palmitoylation-dependent CD95-mediated cell death by targeting acyl protein thioesterases 1 and 2 in CLL. Blood 125, 2948-2957. doi: 10.1182/blood-2014-07586511

Bijlmakers, M. J. (2009). Protein acylation and localization in T cell signaling (Review). Mol. Membr. Biol. 26, 93-103. doi: 10.1080/09687680802650481

Bijlmakers, M. J., and Marsh, M. (2003). The on-off story of protein palmitoylation. Trends Cell Biol. 13, 32-42. doi: 10.1016/s0962-8924(02)000089

Blanpain, C., Wittamer, V., Vanderwinden, J. M., Boom, A., Renneboog, B., Lee, B., et al. (2001). Palmitoylation of CCR5 is critical for receptor trafficking and efficient activation of intracellular signaling pathways. J. Biol. Chem. 276, 23795-23804. doi: 10.1074/jbc.M100583200

Blaskovic, S., Blanc, M., and van der Goot, F. G. (2013). What does S-palmitoylation do to membrane proteins? FEBS J. 280, 2766-2774. doi: 10.1111/febs.12263

Borzecka-Solarz, K., Dembinska, J., Hromada-Judycka, A., Traczyk, G., Ciesielska, A., Ziemlinska, E., et al. (2017). Association of Lyn kinase with membrane rafts determines its negative influence on LPS-induced signaling. Mol. Biol. Cell 28, 1147-1159. doi: 10.1091/mbc.E16-090632

Bournazos, S., Hart, S. P., Chamberlain, L. H., Glennie, M. J., and Dransfield, I. (2009). Association of FcgammaRIIa (CD32a) with lipid rafts regulates ligand binding activity. J. Immunol. 182, 8026-8036. doi: 10.4049/jimmunol.0900107

Bradfield, P. F., Scheiermann, C., Nourshargh, S., Ody, C., Luscinskas, F. W., Rainger, G. E., et al. (2007). JAM-C regulates unidirectional monocyte transendothelial migration in inflammation. Blood 110, 2545-2555. doi: 10. 1182/blood-2007-03078733

Brdicka, T., Imrich, M., Angelisova, P., Brdickova, N., Horvath, O., Spicka, J., et al. (2002). Non-T cell activation linker (NTAL): a transmembrane adaptor protein involved in immunoreceptor signaling. J. Exp. Med. 196, 1617-1626. doi: $10.1084 /$ jem. 20021405

Brigidi, G. S., and Bamji, S. X. (2013). Detection of protein palmitoylation in cultured hippocampal neurons by immunoprecipitation and acyl-biotin exchange (ABE). J. Vis. Exp. 72:50031. doi: 10.3791/50031

Camp, L. A., and Hofmann, S. L. (1993). Purification and properties of a palmitoylprotein thioesterase that cleaves palmitate from H-Ras. J. Biol. Chem. 268, 22566-22574.
Chakrabandhu, K., Herincs, Z., Huault, S., Dost, B., Peng, L., Conchonaud, F., et al. (2007). Palmitoylation is required for efficient Fas cell death signaling. EMBO J. 26, 209-220. doi: 10.1038/sj.emboj.7601456

Charrin, S., Manie, S., Oualid, M., Billard, M., Boucheix, C., and Rubinstein, E. (2002). Differential stability of tetraspanin/tetraspanin interactions: role of palmitoylation. FEBS Lett. 516, 139-144. doi: 10.1016/s0014-5793(02)02522-x

Charron, G., Zhang, M. M., Yount, J. S., Wilson, J., Raghavan, A. S., Shamir, E., et al. (2009). Robust fluorescent detection of protein fatty-acylation with chemical reporters. J. Am. Chem. Soc. 131, 4967-4975. doi: 10.1021/ja810122f

Cherukuri, A., Carter, R. H., Brooks, S., Bornmann, W., Finn, R., Dowd, C. S., et al. (2004). B cell signaling is regulated by induced palmitoylation of CD81. J. Biol. Chem. 279, 31973-31982. doi: 10.1074/jbc.M404410200

Chesarino, N. M., Hach, J. C., Chen, J. L., Zaro, B. W., Rajaram, M. V., Turner, J., et al. (2014). Chemoproteomics reveals Toll-like receptor fatty acylation. $B M C$ Biol. 12:91. doi: 10.1186/s12915-014-009193

Chini, B., and Parenti, M. (2009). G-protein-coupled receptors, cholesterol and palmitoylation: facts about fats. J. Mol. Endocrinol. 42, 371-379. doi: 10.1677/ JME-080114

Cho, E., and Park, M. (2016). Palmitoylation in Alzheimer's disease and other neurodegenerative diseases. Pharmacol. Res. 111, 133-151. doi: 10.1016/j.phrs. 2016.06.008

Clark, K. L., Oelke, A., Johnson, M. E., Eilert, K. D., Simpson, P. C., and Todd, S. C. (2004). CD81 associates with $14-3-3$ in a redox-regulated palmitoylation-dependent manner. J. Biol. Chem. 279, 19401-19406. doi: 10. 1074/jbc.M312626200

Claudinon, J., Gonnord, P., Beslard, E., Marchetti, M., Mitchell, K., Boularan, C., et al. (2009). Palmitoylation of interferon-alpha (IFN-alpha) receptor subunit IFNAR1 is required for the activation of Stat1 and Stat2 by IFN-alpha. J. Biol. Chem. 284, 24328-24340. doi: 10.1074/jbc.M109.021915

Courtney, A. H., Lo, W. L., and Weiss, A. (2018). TCR Signaling: Mechanisms of Initiation and Propagation. Trends Biochem. Sci. 43, 108-123. doi: 10.1016/j. tibs.2017.11.008

Crise, B., and Rose, J. K. (1992). Identification of palmitoylation sites on CD4, the human immunodeficiency virus receptor. J. Biol. Chem. 267, 13593-13597.

Cruz, A. C., Ramaswamy, M., Ouyang, C., Klebanoff, C. A., Sengupta, P., Yamamoto, T. N., et al. (2016). Fas/CD95 prevents autoimmunity independently of lipid raft localization and efficient apoptosis induction. Nat. Commun. 7:13895. doi: 10.1038/ncomms 13895

Davda, D., El Azzouny, M. A., Tom, C. T., Hernandez, J. L., Majmudar, J. D., Kennedy, R. T., et al. (2013). Profiling targets of the irreversible palmitoylation inhibitor 2-bromopalmitate. ACS Chem. Biol. 8, 1912-1917. doi: 10.1021/ cb400380s

Davidson, D., Bakinowski, M., Thomas, M. L., Horejsi, V., and Veillette, A. (2003). Phosphorylation-dependent regulation of T-cell activation by PAG/Cbp, a lipid raft-associated transmembrane adaptor. Mol. Cell Biol. 23, 2017-2028. doi: 10.1128/mcb.23.6.2017-2028.2003

Delandre, C., Penabaz, T. R., Passarelli, A. L., Chapes, S. K., and Clem, R. J. (2009). Mutation of juxtamembrane cysteines in the tetraspanin CD81 affects palmitoylation and alters interaction with other proteins at the cell surface. Exp. Cell Res. 315, 1953-1963. doi: 10.1016/j.yexcr.2009.03.013

Draber, P., Vonkova, I., Stepanek, O., Hrdinka, M., Kucova, M., Skopcova, T., et al. (2011). SCIMP, a transmembrane adaptor protein involved in major histocompatibility complex class II signaling. Mol. Cell Biol. 31, 4550-4562. doi: 10.1128/MCB.058175811

Drisdel, R. C., and Green, W. N. (2004). Labeling and quantifying sites of protein palmitoylation. Biotechniques 36, 276-285. doi: 10.2144/04362RR02

Duncan, J. A., and Gilman, A. G. (1998). A cytoplasmic acyl-protein thioesterase that removes palmitate from G protein alpha subunits and p21(RAS). J. Biol. Chem. 273, 15830-15837. doi: 10.1074/jbc.273.25.15830

Ebsen, H., Lettau, M., Kabelitz, D., and Janssen, O. (2015). Subcellular localization and activation of ADAM proteases in the context of FasL shedding in T lymphocytes. Mol. Immunol. 65, 416-428. doi: 10.1016/j.molimm.2015. 02.008

Fernandez-Hernando, C., Fukata, M., Bernatchez, P. N., Fukata, Y., Lin, M. I., Bredt, D. S., et al. (2006). Identification of Golgi-localized acyl transferases that palmitoylate and regulate endothelial nitric oxide synthase. J. Cell Biol. 174, 369-377. doi: $10.1083 /$ jcb.200601051 
Fessler, M. B., Rudel, L. L., and Brown, J. M. (2009). Toll-like receptor signaling links dietary fatty acids to the metabolic syndrome. Curr. Opin. Lipidol. 20, 379-385. doi: 10.1097/MOL.0b013e32832fa5c4

Filipp, D., Zhang, J., Leung, B. L., Shaw, A., Levin, S. D., Veillette, A., et al. (2003). Regulation of Fyn through translocation of activated Lck into lipid rafts. J. Exp. Med. 197, 1221-1227. doi: 10.1084/jem.20022112

Filippi, M. D., Szczur, K., Harris, C. E., and Berclaz, P. Y. (2007). Rho GTPase Racl is critical for neutrophil migration into the lung. Blood 109, 1257-1264. doi: 10.1182/blood-2006-04017731

Fillatreau, S. (2018). B cells and their cytokine activities implications in human diseases. Clin. Immunol. 186, 26-31. doi: 10.1016/j.clim.2017.07.020

Fitzer-Attas, C. J., Lowry, M., Crowley, M. T., Finn, A. J., Meng, F., DeFranco, A. L., et al. (2000). Fcgamma receptor-mediated phagocytosis in macrophages lacking the Src family tyrosine kinases Hck, Fgr, and Lyn. J. Exp. Med. 191, 669-682. doi: $10.1084 /$ jem.191.4.669

Flaumenhaft, R., and Sim, D. S. (2005). Protein palmitoylation in signal transduction of hematopoietic cells. Hematology 10, 511-519. doi: 10.1080/ 10245330500141507

Forrester, M. T., Hess, D. T., Thompson, J. W., Hultman, R., Moseley, M. A., Stamler, J. S., et al. (2011). Site-specific analysis of protein S-acylation by resin-assisted capture. J. Lipid. Res. 52, 393-398. doi: 10.1194/jlr.D011106

Fredericks, G. J., Hoffmann, F. W., Rose, A. H., Osterheld, H. J., Hess, F. M., Mercier, F., et al. (2014). Stable expression and function of the inositol 1,4,5triphosphate receptor requires palmitoylation by a DHHC6/selenoprotein K complex. Proc. Natl. Acad. Sci. U S A 111, 16478-16483. doi: 10.1073/pnas. 1417176111

Futosi, K., Fodor, S., and Mocsai, A. (2013). Neutrophil cell surface receptors and their intracellular signal transduction pathways. Int. Immunopharmacol. 17, 638-650. doi: 10.1016/j.intimp.2013.06.034

Gadalla, M. R., and Veit, M. (2020). Toward the identification of ZDHHC enzymes required for palmitoylation of viral protein as potential drug targets. Expert. Opin. Drug. Discov. 15, 159-177. doi: 10.1080/17460441.2020.1696306

Gao, X., and Hannoush, R. N. (2014). Single-cell imaging of Wnt palmitoylation by the acyltransferase porcupine. Nat. Chem. Biol. 10, 61-68. doi: 10.1038/ nchembio. 1392

Gonzalez-Navajas, J. M., Lee, J., David, M., and Raz, E. (2012). Immunomodulatory functions of type I interferons. Nat. Rev. Immunol. 12, 125-135. doi: 10.1038/ nri3133

Gordon, D. E., Jang, G. M., Bouhaddou, M., Xu, J., Obernier, K., White, K. M., et al. (2020). A SARS-CoV-2 protein interaction map reveals targets for drug repurposing. Nature 583, 459-468. doi: 10.1038/s41586-020-22862289

Greaves, J., and Chamberlain, L. H. (2007). Palmitoylation-dependent protein sorting. J. Cell Biol. 176, 249-254. doi: 10.1083/jcb.200610151

Gruda, R., Achdout, H., Stern-Ginossar, N., Gazit, R., Betser-Cohen, G., Manaster, I., et al. (2007). Intracellular cysteine residues in the tail of MHC class I proteins are crucial for extracellular recognition by leukocyte Ig-like receptor 1. J. Immunol. 179, 3655-3661. doi: 10.4049/jimmunol.179.6.3655

Gupta, P., Soyombo, A. A., Atashband, A., Wisniewski, K. E., Shelton, J. M., Richardson, J. A., et al. (2001). Disruption of PPT1 or PPT2 causes neuronal ceroid lipofuscinosis in knockout mice. Proc. Natl. Acad. Sci. U S A 98, 13566-13571. doi: 10.1073/pnas.251485198

Hancock, J. F., Paterson, H., and Marshall, C. J. (1990). A polybasic domain or palmitoylation is required in addition to the CAAX motif to localize p21ras to the plasma membrane. Cell 63, 133-139. doi: 10.1016/0092-8674(90)90294-o

Hang, H. C., Geutjes, E. J., Grotenbreg, G., Pollington, A. M., Bijlmakers, M. J., and Ploegh, H. L. (2007). Chemical probes for the rapid detection of Fattyacylated proteins in Mammalian cells. J. Am. Chem. Soc. 129, 2744-2745. doi: 10.1021/ja0685001

Hannoush, R. N., and Arenas-Ramirez, N. (2009). Imaging the lipidome: omegaalkynyl fatty acids for detection and cellular visualization of lipid-modified proteins. ACS Chem. Biol. 4, 581-587. doi: 10.1021/cb900085z

Hansen, A. L., Buchan, G. J., Ruhl, M., Mukai, K., Salvatore, S. R., Ogawa, E., et al. (2018). Nitro-fatty acids are formed in response to virus infection and are potent inhibitors of STING palmitoylation and signaling. Proc. Natl. Acad. Sci. U S A 115, E7768-E7775. doi: 10.1073/pnas.1806239115

Hansen, A. L., Mukai, K., Schopfer, F. J., Taguchi, T., and Holm, C. K. (2019). STING palmitoylation as a therapeutic target. Cell Mol. Immunol. 16, 236-241. doi: 10.1038/s41423-019-0205205
Harder, T., and Kuhn, M. (2000). Selective accumulation of raft-associated membrane protein LAT in T cell receptor signaling assemblies. J. Cell Biol. 151, 199-208. doi: 10.1083/jcb.151.2.199

He, Y., and Linder, M. E. (2009). Differential palmitoylation of the endosomal SNAREs syntaxin 7 and syntaxin 8. J. Lipid. Res. 50, 398-404. doi: 10.1194/jlr. M800360-JLR200

Hellewell, A. L., Foresti, O., Gover, N., Porter, M. Y., and Hewitt, E. W. (2014). Analysis of familial hemophagocytic lymphohistiocytosis type 4 (FHL-4) mutant proteins reveals that $\mathrm{S}$-acylation is required for the function of syntaxin 11 in natural killer cells. PLoS One 9:e98900. doi: 10.1371/journal.pone.0098900

Hewitt, E. W. (2003). The MHC class I antigen presentation pathway: strategies for viral immune evasion. Immunology 110, 163-169. doi: 10.1046/j.1365-2567. 2003.01738.x

Hilger, D., Masureel, M., and Kobilka, B. K. (2018). Structure and dynamics of GPCR signaling complexes. Nat. Struct. Mol. Biol. 25, 4-12. doi: 10.1038/ s41594-017-001117

Hind, L. E., Vincent, W. J., and Huttenlocher, A. (2016). Leading from the Back: The Role of the Uropod in Neutrophil Polarization and Migration. Dev. Cell 38, 161-169. doi: 10.1016/j.devcel.2016.06.031

Horejsi, V. (2004). Transmembrane adaptor proteins in membrane microdomains: important regulators of immunoreceptor signaling. Immunol. Lett. 92, 43-49. doi: 10.1016/j.imlet.2003.10.013

Horejsi, V., Zhang, W., and Schraven, B. (2004). Transmembrane adaptor proteins: organizers of immunoreceptor signalling. Nat. Rev. Immunol. 4, 603-616. doi: $10.1038 /$ nri1414

Hornemann, T. (2015). Palmitoylation and depalmitoylation defects. J. Inher. Metab. Dis. 38, 179-186. doi: 10.1007/s10545-014-97539750

Hundt, M., Tabata, H., Jeon, M. S., Hayashi, K., Tanaka, Y., Krishna, R., et al. (2006). Impaired activation and localization of LAT in anergic T cells as a consequence of a selective palmitoylation defect. Immunity 24, 513-522. doi: 10.1016/j.immuni.2006.03.011

Hur, E. M., Son, M., Lee, O. H., Choi, Y. B., Park, C., Lee, H., et al. (2003). LIME, a novel transmembrane adaptor protein, associates with p56lck and mediates T cell activation. J. Exp. Med. 198, 1463-1473. doi: 10.1084/jem.200 30232

Ivaldi, C., Martin, B. R., Kieffer-Jaquinod, S., Chapel, A., Levade, T., Garin, J., et al. (2012). Proteomic analysis of S-acylated proteins in human B cells reveals palmitoylation of the immune regulators CD20 and CD23. PLoS One 7:e37187. doi: 10.1371/journal.pone.0037187

Ivashkiv, L. B., and Donlin, L. T. (2014). Regulation of type I interferon responses. Nat. Rev. Immunol. 14, 36-49. doi: 10.1038/nri3581

Juneja, V. R., McGuire, K. A., Manguso, R. T., LaFleur, M. W., Collins, N., Haining, W. N., et al. (2017). PD-L1 on tumor cells is sufficient for immune evasion in immunogenic tumors and inhibits CD8 T cell cytotoxicity. J. Exp. Med. 214, 895-904. doi: 10.1084/jem.20160801

Kabouridis, P. S. (2006). Lipid rafts in T cell receptor signalling. Mol. Membr. Biol. 23, 49-57. doi: 10.1080/09687860500453673

Kabouridis, P. S., Magee, A. I., and Ley, S. C. (1997). S-acylation of LCK protein tyrosine kinase is essential for its signalling function in T lymphocytes. EMBO J. 16, 4983-4998. doi: 10.1093/emboj/16.16.4983

Kang, R., Wan, J., Arstikaitis, P., Takahashi, H., Huang, K., Bailey, A. O., et al. (2008). Neural palmitoyl-proteomics reveals dynamic synaptic palmitoylation. Nature 456, 904-909. doi: 10.1038/nature07605

Kara, S., Amon, L., Luhr, J. J., Nimmerjahn, F., Dudziak, D., and Lux, A. (2020). Impact of Plasma Membrane Domains on IgG Fc Receptor Function. Front. Immunol. 11:1320. doi: 10.3389/fimmu.2020.01320

Kim, S. J., Zhang, Z., Sarkar, C., Tsai, P. C., Lee, Y. C., Dye, L., et al. (2008). Palmitoyl protein thioesterase- 1 deficiency impairs synaptic vesicle recycling at nerve terminals, contributing to neuropathology in humans and mice. J. Clin. Invest. 118, 3075-3086. doi: 10.1172/JCI33482

Kim, Y. C., Lee, S. E., Kim, S. K., Jang, H. D., Hwang, I., Jin, S., et al. (2019). Toll-like receptor mediated inflammation requires FASN-dependent MYD88 palmitoylation. Nat. Chem. Biol. 15, 907-916. doi: 10.1038/s41589019-0344340

Ko, P. J., and Dixon, S. J. (2018). Protein palmitoylation and cancer. EMBO Rep. 19:e46666. doi: 10.15252/embr.201846666

Kong, E., Peng, S., Chandra, G., Sarkar, C., Zhang, Z., Bagh, M. B., et al. (2013). Dynamic palmitoylation links cytosol-membrane shuttling of acyl-protein 
thioesterase-1 and acyl-protein thioesterase- 2 with that of proto-oncogene H-ras product and growth-associated protein-43. J. Biol. Chem. 288, 9112-9125. doi: 10.1074/jbc.M112.421073

Korycka, J., Lach, A., Heger, E., Boguslawska, D. M., Wolny, M., Toporkiewicz, M., et al. (2012). Human DHHC proteins: a spotlight on the hidden player of palmitoylation. Eur. J. Cell Biol. 91, 107-117. doi: 10.1016/j.ejcb.2011.09.013

Koster, K. P., and Yoshii, A. (2019). Depalmitoylation by Palmitoyl-Protein Thioesterase 1 in Neuronal Health and Degeneration. Front. Synap. Neurosci. 11:25. doi: 10.3389/fnsyn.2019.00025

Kosugi, A., Hayashi, F., Liddicoat, D. R., Yasuda, K., Saitoh, S., and Hamaoka, T. (2001). A pivotal role of cysteine 3 of Lck tyrosine kinase for localization to glycolipid-enriched microdomains and T cell activation. Immunol. Lett. 76, 133-138. doi: 10.1016/s0165-2478(01)00174172

Kwiatkowska, K., and Ciesielska, A. (2018). Lipid-mediated regulation of proinflammatory responses induced by lipopolysaccharide. Postepy Biochem. 64, 175-182. doi: 10.18388/pb.2018_129

Ladygina, N., Martin, B. R., and Altman, A. (2011). Dynamic palmitoylation and the role of DHHC proteins in T cell activation and anergy. Adv. Immunol. 109, 1-44. doi: 10.1016/B978-0-12-387664-5.000017

Lammermann, T., and Kastenmuller, W. (2019). Concepts of GPCR-controlled navigation in the immune system. Immunol. Rev. 289, 205-231. doi: 10.1111/ imr. 12752

Lancaster, G. I., Langley, K. G., Berglund, N. A., Kammoun, H. L., Reibe, S., Estevez, E., et al. (2018). Evidence that TLR4 Is Not a Receptor for Saturated Fatty Acids but Mediates Lipid-Induced Inflammation by Reprogramming Macrophage Metabolism. Cell. Metab. 27(5):e1095. doi: 10.1016/j.cmet.2018. 03.014

Lee, A. J., and Ashkar, A. A. (2018). The Dual Nature of Type I and Type II Interferons. Front. Immunol. 9:2061. doi: 10.3389/fimmu.2018.02061

Liang, X., Nazarian, A., Erdjument-Bromage, H., Bornmann, W., Tempst, P., and Resh, M. D. (2001). Heterogeneous fatty acylation of Src family kinases with polyunsaturated fatty acids regulates raft localization and signal transduction. J. Biol. Chem. 276, 30987-30994. doi: 10.1074/jbc.M104018200

Lin, D. T., and Conibear, E. (2015). ABHD17 proteins are novel protein depalmitoylases that regulate N-Ras palmitate turnover and subcellular localization. Elife 4:e11306. doi: 10.7554/eLife.11306

Lisman, T. (2018). Platelet-neutrophil interactions as drivers of inflammatory and thrombotic disease. Cell Tissue Res. 371, 567-576. doi: 10.1007/s00441-01727272724

Liu, J., Garcia-Cardena, G., and Sessa, W. C. (1995). Biosynthesis and palmitoylation of endothelial nitric oxide synthase: mutagenesis of palmitoylation sites, cysteines-15 and/or -26, argues against depalmitoylationinduced translocation of the enzyme. Biochemistry 34, 12333-12340. doi: 10.1021/bi00038a029

Liu, J., Garcia-Cardena, G., and Sessa, W. C. (1996). Palmitoylation of endothelial nitric oxide synthase is necessary for optimal stimulated release of nitric oxide: implications for caveolae localization. Biochemistry 35, 13277-13281. doi: 10. 1021/bi961720e

Liu, J., Qian, C., and Cao, X. (2016). Post-Translational Modification Control of Innate Immunity. Immunity 45, 15-30. doi: 10.1016/j.immuni.2016.06.020

Lobo, S., Greentree, W. K., Linder, M. E., and Deschenes, R. J. (2002). Identification of a Ras palmitoyltransferase in Saccharomyces cerevisiae. J. Biol. Chem. 277, 41268-41273. doi: 10.1074/jbc.M206573200

Lu, X., Sicard, R., Jiang, X., Stockus, J. N., McNamara, G., Abdulreda, M., et al. (2015). HGAL localization to cell membrane regulates B-cell receptor signaling. Blood 125, 649-657. doi: 10.1182/blood-2014-04571331

Lu, Y., Zheng, Y., Coyaud, E., Zhang, C., Selvabaskaran, A., Yu, Y., et al. (2019). Palmitoylation of NOD1 and NOD2 is required for bacterial sensing. Science 366, 460-467. doi: 10.1126/science.aau6391

Lupica, C. R., Cass, W. A., Zahniser, N. R., and Dunwiddie, T. V. (1990). Effects of the selective adenosine A2 receptor agonist CGS 21680 on in vitro electrophysiology, cAMP formation and dopamine release in rat hippocampus and striatum. J. Pharmacol. Exp. Ther. 252, 1134-1141.

Ma, Y., Yang, X., Chatterjee, V., Meegan, J. E., and Beard, R. S. Jr. (2019). Role of Neutrophil Extracellular Traps and Vesicles in Regulating Vascular Endothelial Permeability. Front. Immunol. 10:1037. doi: 10.3389/fimmu.2019.01037

Magee, A. I., Gutierrez, L., McKay, I. A., Marshall, C. J., and Hall, A. (1987). Dynamic fatty acylation of p21N-ras. EMBO J. 6, 3353-3357.
Marin, E. P., Derakhshan, B., Lam, T. T., Davalos, A., and Sessa, W. C. (2012). Endothelial cell palmitoylproteomic identifies novel lipid-modified targets and potential substrates for protein acyl transferases. Circ. Res. 110, 1336-1344. doi: 10.1161/CIRCRESAHA.112.269514

Martin, B. R., and Cravatt, B. F. (2009). Large-scale profiling of protein palmitoylation in mammalian cells. Nat. Methods 6, 135-138. doi: 10.1038/ nmeth.1293

Matt, L., Kim, K., Chowdhury, D., and Hell, J. W. (2019). Role of Palmitoylation of Postsynaptic Proteins in Promoting Synaptic Plasticity. Front. Mol. Neurosci. 12:8. doi: 10.3389/fnmol.2019.00008

Meiler, S., Baumer, Y., Huang, Z., Hoffmann, F. W., Fredericks, G. J., Rose, A. H., et al. (2013). Selenoprotein K is required for palmitoylation of CD36 in macrophages: implications in foam cell formation and atherogenesis. J. Leukoc. Biol. 93, 771-780. doi: 10.1189/jlb.1212647

Merrick, B. A., Dhungana, S., Williams, J. G., Aloor, J. J., Peddada, S., Tomer, K. B., et al. (2011). Proteomic profiling of S-acylated macrophage proteins identifies a role for palmitoylation in mitochondrial targeting of phospholipid scramblase 3. Mol. Cell Proteom. 10:M110006007. doi: 10.1074/mcp.M110.006007

Mill, P., Lee, A. W., Fukata, Y., Tsutsumi, R., Fukata, M., Keighren, M., et al. (2009). Palmitoylation regulates epidermal homeostasis and hair follicle differentiation. PLoS Gen. 5:e1000748. doi: 10.1371/journal.pgen.1000748

Mocsai, A., Walzog, B., and Lowell, C. A. (2015). Intracellular signalling during neutrophil recruitment. Cardiovasc. Res. 107, 373-385. doi: 10.1093/cvr/cvv159

Moffett, S., Mouillac, B., Bonin, H., and Bouvier, M. (1993). Altered phosphorylation and desensitization patterns of a human beta 2-adrenergic receptor lacking the palmitoylated Cys341. EMBO J. 12, 349-356.

Molina, T. J., Kishihara, K., Siderovski, D. P., van Ewijk, W., Narendran, A., Timms, E., et al. (1992). Profound block in thymocyte development in mice lacking p56lck. Nature 357, 161-164. doi: 10.1038/357161a0

Morrison, E., Kuropka, B., Kliche, S., Brugger, B., Krause, E., and Freund, C. (2015). Quantitative analysis of the human T cell palmitome. Sci. Rep. 5:11598. doi: 10.1038/srep 11598

Moutin, E., Nikonenko, I., Stefanelli, T., Wirth, A., Ponimaskin, E., De Roo, M., et al. (2017). Palmitoylation of cdc42 Promotes Spine Stabilization and Rescues Spine Density Deficit in a Mouse Model of 22q11.2 Deletion Syndrome. Cereb. Cortex. 27, 3618-3629. doi: 10.1093/cercor/bhw183

Mukai, K., Konno, H., Akiba, T., Uemura, T., Waguri, S., Kobayashi, T., et al. (2016). Activation of STING requires palmitoylation at the Golgi. Nat. Commun. 7:11932. doi: 10.1038/ncomms11932

Nagaleekar, V. K., Diehl, S. A., Juncadella, I., Charland, C., Muthusamy, N., Eaton, S., et al. (2008). IP3 receptor-mediated Ca2+ release in naive CD4 T cells dictates their cytokine program. J. Immunol. 181, 8315-8322. doi: 10.4049/jimmunol. 181.12 .8315

Navarro-Lerida, I., Sanchez-Perales, S., Calvo, M., Rentero, C., Zheng, Y., Enrich, C., et al. (2012). A palmitoylation switch mechanism regulates Racl function and membrane organization. EMBO J. 31, 534-551. doi: 10.1038/emboj.201 1.446

Norton, R. L., Fredericks, G. J., Huang, Z., Fay, J. D., Hoffmann, F. W., and Hoffmann, P. R. (2017). Selenoprotein K regulation of palmitoylation and calpain cleavage of ASAP2 is required for efficient FcgammaRmediated phagocytosis. J. Leukoc. Biol. 101, 439-448. doi: 10.1189/jlb.2A0316156RR

O'Brien, C. D., Lim, P., Sun, J., and Albelda, S. M. (2003). PECAM-1-dependent neutrophil transmigration is independent of monolayer PECAM-1 signaling or localization. Blood 101, 2816-2825. doi: 10.1182/blood-2002-082396

Palacios, E. H., and Weiss, A. (2004). Function of the Src-family kinases, Lck and Fyn, in T-cell development and activation. Oncogene 23, 7990-8000. doi: 10.1038/sj.onc. 1208074

Pang, D. J., Hayday, A. C., and Bijlmakers, M. J. (2007). CD8 Raft localization is induced by its assembly into CD8alpha beta heterodimers, Not CD8alpha alpha homodimers. J. Biol. Chem. 282, 13884-13894. doi: 10.1074/jbc.M701027200

Pegram, H. J., Andrews, D. M., Smyth, M. J., Darcy, P. K., and Kershaw, M. H. (2011). Activating and inhibitory receptors of natural killer cells. Immunol. Cell Biol. 89, 216-224. doi: 10.1038/icb.2010.78

Percherancier, Y., Planchenault, T., Valenzuela-Fernandez, A., Virelizier, J. L., Arenzana-Seisdedos, F., and Bachelerie, F. (2001). Palmitoylation-dependent control of degradation, life span, and membrane expression of the CCR5 receptor. J. Biol. Chem. 276, 31936-31944. doi: 10.1074/jbc.M104013200 
Petit, C. M., Chouljenko, V. N., Iyer, A., Colgrove, R., Farzan, M., Knipe, D. M., et al. (2007). Palmitoylation of the cysteine-rich endodomain of the SARScoronavirus spike glycoprotein is important for spike-mediated cell fusion. Virology 360, 264-274. doi: 10.1016/j.virol.2006.10.034

Poggi, M., Kara, I., Brunel, J. M., Landrier, J. F., Govers, R., Bonardo, B., et al. (2013). Palmitoylation of TNF alpha is involved in the regulation of TNF receptor 1 signalling. Biochim. Biophys. Acta 1833, 602-612. doi: 10.1016/j. bbamcr.2012.11.009

Posevitz-Fejfar, A., Smida, M., Kliche, S., Hartig, R., Schraven, B., and Lindquist, J. A. (2008). A displaced PAG enhances proximal signaling and SDF-1-induced T cell migration. Eur. J. Immunol. 38, 250-259. doi: 10.1002/eji.200636664

Qanbar, R., and Bouvier, M. (2003). Role of palmitoylation/depalmitoylation reactions in G-protein-coupled receptor function. Pharmacol. Ther. 97, 1-33. doi: 10.1016/s0163-7258(02)00300305

Quast, T., Eppler, F., Semmling, V., Schild, C., Homsi, Y., Levy, S., et al. (2011). CD81 is essential for the formation of membrane protrusions and regulates Rac1-activation in adhesion-dependent immune cell migration. Blood 118, 1818-1827. doi: 10.1182/blood-2010-12326595

Quinn, B. J., Welch, E. J., Kim, A. C., Lokuta, M. A., Huttenlocher, A., Khan, A. A., et al. (2009). Erythrocyte scaffolding protein p55/MPP1 functions as an essential regulator of neutrophil polarity. Proc. Natl. Acad. Sci. U S A 106, 19842-19847. doi: 10.1073/pnas.0906761106

Rana, M. S., Lee, C. J., and Banerjee, A. (2019). The molecular mechanism of DHHC protein acyltransferases. Biochem. Soc. Trans. 47, 157-167. doi: 10.1042/ BST20180429

Resh, M. D. (1994). Myristylation and palmitylation of Src family members: the fats of the matter. Cell 76, 411-413. doi: 10.1016/0092-8674(94)90104-x

Resh, M. D. (2006a). Palmitoylation of ligands, receptors, and intracellular signaling molecules. Sci. Stke 2006:re14. doi: 10.1126/stke.3592006re14

Resh, M. D. (2006b). Use of analogs and inhibitors to study the functional significance of protein palmitoylation. Methods 40, 191-197. doi: 10.1016/j. ymeth.2006.04.013

Resh, M. D. (2013). Covalent lipid modifications of proteins. Curr. Biol. 23, R431-R435. doi: 10.1016/j.cub.2013.04.024

Rodolfo, M., Salvi, C., Bassi, C., Rovetta, G., Melani, C., Colombo, M. P., et al. (1991). Adjuvant adoptive immunotherapy with IL2 and lymphocytes from tumor-bearing mice: in vitro tumor-stimulated lymphocytes are more effective than LAK cells. Nat. Immun. Cell Growth Regul. 10, 308-319.

Romero-Camarero, I., Jiang, X., Natkunam, Y., Lu, X., Vicente-Duenas, C., Gonzalez-Herrero, I., et al. (2013). Germinal centre protein HGAL promotes lymphoid hyperplasia and amyloidosis via BCR-mediated Syk activation. Nat. Commun. 4:1338. doi: 10.1038/ncomms 2334

Ross, E. M. (1995). Protein modification. Palmitoylation in G-protein signaling pathways. Curr. Biol. 5, 107-109. doi: 10.1016/s0960-9822(95)0002621

Rossin, A., Durivault, J., Chakhtoura-Feghali, T., Lounnas, N., Gagnoux-Palacios, L., and Hueber, A. O. (2015). Fas palmitoylation by the palmitoyl acyltransferase DHHC7 regulates Fas stability. Cell Death Differ. 22, 643-653. doi: 10.1038/cdd. 2014.153

Roth, A. F., Feng, Y., Chen, L., and Davis, N. G. (2002). The yeast DHHC cysteinerich domain protein Akrlp is a palmitoyl transferase. J. Cell Biol. 159, 23-28. doi: $10.1083 /$ jcb.200206120

Rusch, M., Zimmermann, T. J., Burger, M., Dekker, F. J., Gormer, K., Triola, G., et al. (2011). Identification of acyl protein thioesterases 1 and 2 as the cellular targets of the Ras-signaling modulators palmostatin B and M. Angew. Chem. Int. Ed. Engl. 50, 9838-9842. doi: 10.1002/anie.201102967

Salaun, C., Greaves, J., and Chamberlain, L. H. (2010). The intracellular dynamic of protein palmitoylation. J. Cell Biol. 191, 1229-1238. doi: 10.1083/jcb.2010 08160

Sardjono, C. T., Harbour, S. N., Yip, J. C., Paddock, C., Tridandapani, S., Newman, P. J., et al. (2006). Palmitoylation at Cys595 is essential for PECAM1 localisation into membrane microdomains and for efficient PECAM-1mediated cytoprotection. Thromb. Haem. 96, 756-766.

Satou, M., Nishi, Y., Yoh, J., Hattori, Y., and Sugimoto, H. (2010). Identification and characterization of acyl-protein thioesterase 1/lysophospholipase I as a ghrelin deacylation/lysophospholipid hydrolyzing enzyme in fetal bovine serum and conditioned medium. Endocrinology 151, 4765-4775. doi: 10.1210/ en.20102412
Scheiermann, C., Colom, B., Meda, P., Patel, N. S., Voisin, M. B., Marrelli, A., et al. (2009). Junctional adhesion molecule-C mediates leukocyte infiltration in response to ischemia reperfusion injury. Arteriosc. Thromb. Vasc. Biol. 29, 1509-1515. doi: 10.1161/ATVBAHA.109.187559

Schmidt, M. F., and Schlesinger, M. J. (1979). Fatty acid binding to vesicular stomatitis virus glycoprotein: a new type of post-translational modification of the viral glycoprotein. Cell 17, 813-819. doi: 10.1016/0092-8674(79) 9032190320

Sessa, W. C., Barber, C. M., and Lynch, K. R. (1993). Mutation of N-myristoylation site converts endothelial cell nitric oxide synthase from a membrane to a cytosolic protein. Circ. Res. 72, 921-924. doi: 10.1161/01.res.72.4.921

Shogomori, H., Hammond, A. T., Ostermeyer-Fay, A. G., Barr, D. J., Feigenson, G. W., London, E., et al. (2005). Palmitoylation and intracellular domain interactions both contribute to raft targeting of linker for activation of $\mathrm{T}$ cells. J. Biol. Chem. 280, 18931-18942. doi: 10.1074/jbc.M500247200

Shu, X., Keller, T. C. T., Begandt, D., Butcher, J. T., Biwer, L., Keller, A. S., et al. (2015). Endothelial nitric oxide synthase in the microcirculation. Cell Mol. Life Sci. 72, 4561-4575. doi: 10.1007/s00018-015-20212020

Silverstein, R. L., and Febbraio, M. (2009). CD36, a scavenger receptor involved in immunity, metabolism, angiogenesis, and behavior. Sci. Signal 2:re3. doi: 10.1126/scisignal.272re3

Sim, D. S., Dilks, J. R., and Flaumenhaft, R. (2007). Platelets possess and require an active protein palmitoylation pathway for agonist-mediated activation and in vivo thrombus formation. Arterios. Thromb. Vasc. Biol. 27, 1478-1485. doi: 10.1161/ATVBAHA.106.139287

Skubitz, K. M., Campbell, K. D., Iida, J., and Skubitz, A. P. (1996). CD63 associates with tyrosine kinase activity and CD11/CD18, and transmits an activation signal in neutrophils. J. Immunol. 157, 3617-3626.

Sobocinska, J., Roszczenko-Jasinska, P., Ciesielska, A., and Kwiatkowska, K. (2017). Protein Palmitoylation and Its Role in Bacterial and Viral Infections. Front. Immunol. 8:2003. doi: 10.3389/fimmu.2017.02003

Sobocinska, J., Roszczenko-Jasinska, P., Zareba-Koziol, M., Hromada-Judycka, A., Matveichuk, O. V., Traczyk, G., et al. (2018). Lipopolysaccharide Upregulates Palmitoylated Enzymes of the Phosphatidylinositol Cycle: An Insight from Proteomic Studies. Mol. Cell Proteom. 17, 233-254. doi: 10.1074/mcp.RA117. 000050

Song, S. P., Hennig, A., Schubert, K., Markwart, R., Schmidt, P., Prior, I. A., et al. (2013). Ras palmitoylation is necessary for $\mathrm{N}$-Ras activation and signal propagation in growth factor signalling. Biochem. J. 454, 323-332. doi: 10.1042/ BJ20121799

Soyombo, A. A., and Hofmann, S. L. (1997). Molecular cloning and expression of palmitoyl-protein thioesterase 2 (PPT2), a homolog of lysosomal palmitoylprotein thioesterase with a distinct substrate specificity. J. Biol. Chem. 272, 27456-27463. doi: 10.1074/jbc.272.43.27456

Spence, J. S., He, R., Hoffmann, H. H., Das, T., Thinon, E., Rice, C. M., et al. (2019). IFITM3 directly engages and shuttles incoming virus particles to lysosomes. Nat. Chem. Biol. 15, 259-268. doi: 10.1038/s41589-018-0213212

Steinman, R. M., and Banchereau, J. (2007). Taking dendritic cells into medicine. Nature 449, 419-426. doi: 10.1038/nature06175

Stoffel, R. H., Inglese, J., Macrae, A. D., Lefkowitz, R. J., and Premont, R. T. (1998). Palmitoylation increases the kinase activity of the G protein-coupled receptor kinase. GRK6. Biochemistry 37, 16053-16059. doi: 10.1021/bi981432d

Suda, T., Hashimoto, H., Tanaka, M., Ochi, T., and Nagata, S. (1997). Membrane Fas ligand kills human peripheral blood T lymphocytes, and soluble Fas ligand blocks the killing. J. Exp. Med. 186, 2045-2050. doi: 10.1084/jem.186.12. 2045

Sun, C. X., Downey, G. P., Zhu, F., Koh, A. L., Thang, H., and Glogauer, M. (2004). Rac1 is the small GTPase responsible for regulating the neutrophil chemotaxis compass. Blood 104, 3758-3765. doi: 10.1182/blood-2004-030781

Swarthout, J. T., Lobo, S., Farh, L., Croke, M. R., Greentree, W. K., Deschenes, R. J., et al. (2005). DHHC9 and GCP16 constitute a human protein fatty acyltransferase with specificity for H- and N-Ras. J. Biol. Chem. 280, 3114131148. doi: 10.1074/jbc.M504113200

Szczur, K., Xu, H., Atkinson, S., Zheng, Y., and Filippi, M. D. (2006). Rho GTPase CDC42 regulates directionality and random movement via distinct MAPK pathways in neutrophils. Blood 108, 4205-4213. doi: 10.1182/blood2006-03013789 
Tabaczar, S., Czogalla, A., Podkalicka, J., Biernatowska, A., and Sikorski, A. F. (2017). Protein palmitoylation: Palmitoyltransferases and their specificity. Exp. Biol. Med. 242, 1150-1157. doi: 10.1177/1535370217707732

Tanimura, N., Saitoh, S., Kawano, S., Kosugi, A., and Miyake, K. (2006). Palmitoylation of LAT contributes to its subcellular localization and stability. Biochem. Biophys. Res. Commun. 341, 1177-1183. doi: 10.1016/j.bbrc.2006. 01.076

Termini, C. M., Cotter, M. L., Marjon, K. D., Buranda, T., Lidke, K. A., and Gillette, J. M. (2014). The membrane scaffold CD82 regulates cell adhesion by altering alpha4 integrin stability and molecular density. Mol. Biol. Cell 25, 1560-1573. doi: 10.1091/mbc.E13-110660

Tsai, F. D., Wynne, J. P., Ahearn, I. M., and Philips, M. R. (2014). Metabolic labeling of Ras with tritiated palmitate to monitor palmitoylation and depalmitoylation. Methods Mol. Biol. 1120, 33-41. doi: 10.1007/978-1-62703-791-4_3

Tsygankov, A. Y., Mahajan, S., Fincke, J. E., and Bolen, J. B. (1996). Specific association of tyrosine-phosphorylated c-Cbl with Fyn tyrosine kinase in T cells. J. Biol. Chem. 271, 27130-27137. doi: 10.1074/jbc.271.43.27130

Tuttle, D. L., Harrison, J. K., Anders, C., Sleasman, J. W., and Goodenow, M. M. (1998). Expression of CCR5 increases during monocyte differentiation and directly mediates macrophage susceptibility to infection by human immunodeficiency virus type 1. J. Virol. 72, 4962-4969. doi: 10.1128/JVI.72.6. 4962-4969.1998

Utsumi, T., Takeshige, T., Tanaka, K., Takami, K., Kira, Y., Klostergaard, J., et al. (2001). Transmembrane TNF (pro-TNF) is palmitoylated. FEBS Lett. 500, 1-6. doi: 10.1016/s0014-5793(01)02576-5

van Hemert, M. J., Steensma, H. Y., and van Heusden, G. P. (2001). 14-3-3 proteins: key regulators of cell division, signalling and apoptosis. Bioessays 23, 936-946. doi: 10.1002/bies.1134

Verardi, R., Kim, J. S., Ghirlando, R., and Banerjee, A. (2017). Structural Basis for Substrate Recognition by the Ankyrin Repeat Domain of Human DHHC17 Palmitoyltransferase. Structure 25(9):e1336. doi: 10.1016/j.str.2017.06.018

Verma, S., Hoffmann, F. W., Kumar, M., Huang, Z., Roe, K., Nguyen-Wu, E., et al. (2011). Selenoprotein K knockout mice exhibit deficient calcium flux in immune cells and impaired immune responses. J. Immunol. 186, 2127-2137. doi: 10.4049/jimmunol.1002878

Vogel, K., and Roche, P. A. (1999). SNAP-23 and SNAP-25 are palmitoylated in vivo. Biochem. Biophys. Res. Commun. 258, 407-410. doi: 10.1006/bbrc. 1999. 0652

Vogler, O., Barcelo, J. M., Ribas, C., and Escriba, P. V. (2008). Membrane interactions of $\mathrm{G}$ proteins and other related proteins. Biochim. Biophys. Acta 1778, 1640-1652. doi: 10.1016/j.bbamem.2008.03.008

Volpe, E., Sambucci, M., Battistini, L., and Borsellino, G. (2016). Fas-Fas Ligand: Checkpoint of T Cell Functions in Multiple Sclerosis. Front. Immunol. 7:382. doi: 10.3389/fimmu.2016.00382

Wan, J., Roth, A. F., Bailey, A. O., and Davis, N. G. (2007). Palmitoylated proteins: purification and identification. Nat. Protoc. 2, 1573-1584. doi: 10.1038/nprot. 2007.225

Wang, J., Hao, J. W., Wang, X., Guo, H., Sun, H. H., Lai, X. Y., et al. (2019). DHHC4 and DHHC5 Facilitate Fatty Acid Uptake by Palmitoylating and Targeting CD36 to the Plasma Membrane. Cell Rep. 26(1):e205. doi: 10.1016/j.celrep.2018. 12.022

Wang, X., Iyer, A., Lyons, A. B., Korner, H., and Wei, W. (2019). Emerging Roles for G-protein Coupled Receptors in Development and Activation of Macrophages. Front. Immunol. 10:2031. doi: 10.3389/fimmu.2019.02031

Webb, Y., Hermida-Matsumoto, L., and Resh, M. D. (2000). Inhibition of protein palmitoylation, raft localization, and $\mathrm{T}$ cell signaling by 2-bromopalmitate and polyunsaturated fatty acids. J. Biol. Chem. 275, 261-270. doi: 10.1074/jbc.275. 1.261

Wedegaertner, P. B., Chu, D. H., Wilson, P. T., Levis, M. J., and Bourne, H. R. (1993). Palmitoylation is required for signaling functions and membrane attachment of Gq alpha and Gs alpha. J. Biol. Chem. 268, 25001-25008.
Wee, J. L., Schulze, K. E., Jones, E. L., Yeung, L., Cheng, Q., Pereira, C. F., et al. (2015). Tetraspanin CD37 Regulates beta2 Integrin-Mediated Adhesion and Migration in Neutrophils. J. Immunol. 195, 5770-5779. doi: 10.4049/jimmunol. 1402414

Wensveen, F. M., Jelencic, V., and Polic, B. (2018). NKG2D: A Master Regulator of Immune Cell Responsiveness. Front. Immunol. 9:441. doi: 10.3389/fimmu.2018. 00441

Wolven, A., Okamura, H., Rosenblatt, Y., and Resh, M. D. (1997). Palmitoylation of p59fyn is reversible and sufficient for plasma membrane association. Mol. Biol. Cell 8, 1159-1173. doi: 10.1091/mbc.8.6.1159

Won, S. J., Cheung See Kit, M., and Martin, B. R. (2018). Protein depalmitoylases. Crit. Rev. Biochem. Mol. Biol. 53, 83-98. doi: 10.1080/10409238.2017.14 09191

Yadav, R., Larbi, K. Y., Young, R. E., and Nourshargh, S. (2003). Migration of leukocytes through the vessel wall and beyond. Thromb. Haem. 90, 598-606. doi: 10.1160/TH03-040220

Yang, X., Claas, C., Kraeft, S. K., Chen, L. B., Wang, Z., Kreidberg, J. A., et al. (2002). Palmitoylation of tetraspanin proteins: modulation of CD151 lateral interactions, subcellular distribution, and integrin-dependent cell morphology. Mol. Biol. Cell 13, 767-781. doi: 10.1091/mbc.01-050275

Yao, H., Lan, J., Li, C., Shi, H., Brosseau, J. P., Wang, H., et al. (2019). Inhibiting PD-L1 palmitoylation enhances T-cell immune responses against tumours. Nat. Biomed. Eng. 3, 306-317. doi: 10.1038/s41551-019-0375376

Yasuda, K., Nagafuku, M., Shima, T., Okada, M., Yagi, T., Yamada, T., et al. (2002). Cutting edge: Fyn is essential for tyrosine phosphorylation of Csk-binding protein/phosphoprotein associated with glycolipid-enriched microdomains in lipid rafts in resting T cells. J. Immunol. 169, 2813-2817. doi: 10.4049/jimmunol. 169.6.2813

Yeung, L., Hickey, M. J., and Wright, M. D. (2018). The Many and Varied Roles of Tetraspanins in Immune Cell Recruitment and Migration. Front. Immunol. 9:1644. doi: 10.3389/fimmu.2018.01644

Yount, J. S., Moltedo, B., Yang, Y. Y., Charron, G., Moran, T. M., Lopez, C. B., et al. (2010). Palmitoylome profiling reveals S-palmitoylation-dependent antiviral activity of IFITM3. Nat. Chem. Biol. 6, 610-614. doi: 10.1038/nchembio.405

Yount, J. S., Zhang, M. M., and Hang, H. C. (2013). Emerging roles for protein S-palmitoylation in immunity from chemical proteomics. Curr. Opin. Chem. Biol. 17, 27-33. doi: 10.1016/j.cbpa.2012.11.008

Yurchak, L. K., and Sefton, B. M. (1995). Palmitoylation of either Cys-3 or Cys-5 is required for the biological activity of the Lck tyrosine protein kinase. Mol. Cell Biol. 15, 6914-6922. doi: 10.1128/mcb.15.12.6914

Zeidman, R., Buckland, G., Cebecauer, M., Eissmann, P., Davis, D. M., and Magee, A. I. (2011). DHHC2 is a protein S-acyltransferase for Lck. Mol. Membr. Biol. 28, 473-486. doi: 10.3109/09687688.2011.630682

Zhang, W., Irvin, B. J., Trible, R. P., Abraham, R. T., and Samelson, L. E. (1999). Functional analysis of LAT in TCR-mediated signaling pathways using a LATdeficient Jurkat cell line. Int. Immunol. 11, 943-950. doi: 10.1093/intimm/11. 6.943

Zingler, P., Sarchen, V., Glatter, T., Caning, L., Saggau, C., Kathayat, R. S., et al. (2019). Palmitoylation is required for TNF-R1 signaling. Cell Commun. Sign. 17:90. doi: 10.1186/s12964-019-0405408

Conflict of Interest: The authors declare that the research was conducted in the absence of any commercial or financial relationships that could be construed as a potential conflict of interest.

Copyright (c) 2020 Yang, Chatterjee, Ma, Zheng and Yuan. This is an open-access article distributed under the terms of the Creative Commons Attribution License (CC BY). The use, distribution or reproduction in other forums is permitted, provided the original author(s) and the copyright owner(s) are credited and that the original publication in this journal is cited, in accordance with accepted academic practice. No use, distribution or reproduction is permitted which does not comply with these terms. 